\title{
An extension of a theorem of Euler
}

by

Noriko Hirata-Kohno (Tokyo), Shanta Laishram (Mumbai), T. N. Shorey (Mumbai), and R. TiJdeman (Leiden)

1. Introduction. The theorem of Euler ([Eul80], cf. [Mor69, pp. 21-22], [MS03]) referred to in the title of this paper is that a product of four terms in arithmetic progression is never a square. Let $n, d, k \geq 2$ and $y$ be positive integers such that $\operatorname{gcd}(n, d)=1$. We consider the equation

$$
n(n+d) \cdots(n+(k-1) d)=y^{2}
$$

in $n, d, k$ and $y$. It has infinitely many solutions when $k=2$ or 3 . A wellknown conjecture states that (1) with $k \geq 4$ is not possible. We claim

Theorem 1. Equation (1) with $4 \leq k \leq 109$ is not possible.

By Euler, Theorem 1 is valid when $k=4$. The case when $k=5$ is due to Obláth [Obl50]. Independently of the authors, Bennett, Bruin, Gyôry and Hajdu [BBGH06] proved that (1) with $6 \leq k \leq 11$ does not hold. Theorem 1 has been confirmed by Erdôs [Erd39] and Rigge [Rig39], independently of each other, when $d=1$.

Theorem 1 is derived from a more general result and we introduce some notation for stating this. For an integer $\nu>1$, we denote by $P(\nu)$ the greatest prime factor of $\nu$ and we put $P(1)=1$. Let $b$ be a squarefree positive integer such that $P(b) \leq k$. We consider a more general equation than (1), namely

$$
n(n+d) \cdots(n+(k-1) d)=b y^{2} .
$$

We write

$$
n+i d=a_{i} x_{i}^{2} \quad \text { for } 0 \leq i<k
$$

where $a_{i}$ are squarefree integers such that $P\left(a_{i}\right) \leq \max (P(b), k-1)$ and $x_{i}$ are positive integers. Every solution to $(2)$ yields a $k$-tuple $\left(a_{0}, a_{1}, \ldots, a_{k-1}\right)$. We rewrite (2) as

$$
m(m-d) \cdots(m-(k-1) d)=b y^{2}, \quad m=n+(k-1) d .
$$

2000 Mathematics Subject Classification: Primary 11D61.

Key words and phrases: Euler, Diophantine equations, squares, Legendre symbol. 
Equation (4) is called the mirror image of (2). The corresponding $k$-tuple $\left(a_{k-1}, a_{k-2}, \ldots, a_{0}\right)$ is called the mirror image of $\left(a_{0}, a_{1}, \ldots, a_{k-1}\right)$.

Let $P(b)<k$. Erdôs and Selfridge [ES75] proved that (2) with $d=1$ never holds under the assumption that the left-hand side of $(2)$ is divisible by a prime greater than or equal to $k$. The result does not hold unconditionally. As mentioned above, equation (2) with $k=2,3$ and $b=1$ has infinitely many solutions. This is also the case when $k=4$ and $b=6$; see Tijdeman [Tij89]. On the other hand, equation (2) with $k=4$ and $b \neq 6$ does not hold. We consider (2) with $d>1$ and $k \geq 5$. We prove

Theorem 2. Equation (2) with $d>1, P(b)<k$ and $5 \leq k \leq 100$ implies that $\left(a_{0}, a_{1}, \ldots, a_{k-1}\right)$ is among the following tuples or their mirror images:

$$
\begin{aligned}
& k=8:(2,3,1,5,6,7,2,1),(3,1,5,6,7,2,1,10) \\
& k=9:(2,3,1,5,6,7,2,1,10) \\
& k=14:(3,1,5,6,7,2,1,10,11,3,13,14,15,1) ; \\
& k=24:(5,6,7,2,1,10,11,3,13,14,15,1,17,2,19,5,21,22,23,6,1,26,3,7) .
\end{aligned}
$$

Theorem 2 with $k=5$ is due to Mukhopadhyay and Shorey [MS03]. Initially, Bennett, Bruin, Győry, Hajdu [BBGH06] and Hirata-Kohno, Shorey (unpublished), independently, proved Theorem 2 with $k=6$ and $\left(a_{0}, a_{1}, \ldots, a_{5}\right) \neq(1,2,3,1,5,6),(6,5,1,3,2,1)$. Next, Bennett, Bruin, Győry and Hajdu [BBGH06] removed the assumption on $\left(a_{0}, a_{1}, \ldots, a_{5}\right)$ in the above result. Thus (2) with $k=6$ does not hold and we shall refer to it as the case $k=6$. Bennett, Bruin, Gyóry and Hajdu [BBGH06], independently of us, showed that (2) with $7 \leq k \leq 11$ and $P(b) \leq 5$ is not possible. This is now a special case of Theorem 2 .

Let $P(b)=k$. Then we have no new result on (2) with $k=5$. For $k \geq 7$, we prove

TheOREM 3. Equation (2) with $d>1, P(b)=k$ and $7 \leq k \leq 100$ implies that $\left(a_{0}, a_{1}, \ldots, a_{k-1}\right)$ is among the following tuples or their mirror images:

$$
\begin{aligned}
k=7: \quad & (2,3,1,5,6,7,2),(3,1,5,6,7,2,1),(1,5,6,7,2,1,10) ; \\
k=13: & (3,1,5,6,7,2,1,10,11,3,13,14,15), \\
& (1,5,6,7,2,1,10,11,3,13,14,15,1) ; \\
k=19: & (1,5,6,7,2,1,10,11,3,13,14,15,1,17,2,19,5,21,22) ; \\
k=23: & (5,6,7,2,1,10,11,3,13,14,15,1,17,2,19,5,21,22,23,6,1,26,3), \\
& (6,7,2,1,10,11,3,13,14,15,1,17,2,19,5,21,22,23,6,1,26,3,7) .
\end{aligned}
$$

It has been conjectured that (2) with $k \geq 5$ never holds. Granville (unpublished) showed that $k$ is bounded by an absolute constant whenever the $a b c$-conjecture holds; see Laishram [Lai04] for a proof. For the convenience of the proofs, we consider Theorems 2 and 3 together. Therefore we formulate 
THEOREM 4. Let $d>1, P(b) \leq k$ and $5 \leq k \leq 100$. Suppose that $k \neq 5$ if $P(b)=k$. Then (2) does not hold except for the $\left(a_{0}, a_{1}, \ldots, a_{k-1}\right)$ among (5), (6) and their mirror images.

It is clear that Theorem 4 implies Theorems 2 and 3 . In fact the proof of Theorem 4 provides a method for solving (2) for any given value of $k$ unless $\left(a_{0}, a_{1}, \ldots, a_{k-1}\right)$ is given by (5), (6) and their mirror images. This is a new and useful feature of the paper. We have restricted $k$ up to 100 for keeping the computational load under control. It is an open problem to solve (2) for an infinite sequence of values of $k$. A solution to this problem may be an important contribution towards the conjecture stated just after Theorem 3 . Theorem 4 has been applied in [LS] to show that (2) with $k \geq 6$ implies that $d>10^{10}$. For more applications, see [LS].

Now we give a sketch of the proof of Theorem 4 . Let the assumptions of Theorem 4 be satisfied. Assume (2) such that $\left(a_{0}, a_{1}, \ldots, a_{k-1}\right)$ is not among (5), (6) or their mirror images. As already stated, the cases $k=5$ and $k=6$ have already been solved in [MS03] and [BBGH06]. Therefore we suppose that $k \geq 7$. Further it suffices to assume that $k$ is prime and we proceed inductively on $k$. Let $k$ be given. Then we choose a suitable pair $\left(q_{1}, q_{2}\right)$ of distinct primes $\leq k$ such that

$$
\left(\frac{p}{q_{1}}\right)=\left(\frac{p}{q_{2}}\right)
$$

for small primes $p$. For example, when $k=29$, we take $\left(q_{1}, q_{2}\right)=(19,29)$ so that the above relation holds with $p=2,3,5,7$. We show that $q_{1} \nmid d$ and $q_{2} \nmid d$ (see Lemma 8). Assume $q_{1} \mid d$ or $q_{2} \mid d$. Then we find two primes $Q_{1}$ and $Q_{2}$ such that $Q_{1} \mid d$ or $Q_{2} \mid d$ whenever $k \geq 29$ (see Lemma 7). Now we arrive at a contradiction by a counting argument using (9) and Lemmas 1,2 . Hence $q_{1} \nmid d$ and $q_{2} \nmid d$ but this is excluded by Lemma 6 , the proof of which depends on Lemma 5. In fact, we need to apply it repeatedly for $k>11$.

In the case $k=6$, Bennett, Bruin, Gyốry and Hajdu [BBGH06] solved the cases $\left(a_{0}, a_{1}, \ldots, a_{5}\right) \in\{(1,2,3,1,5,6),(6,5,1,3,2,1)\}$ by using explicit Chabauty techniques due to Bruin and Flynn [BF05]. These cases appear to be similar to our exceptional cases (5) and (6) where we have, in fact, more freedom in the sense that there are at least 7 curves where we may consider applying the Chabauty method. Finally we remark that it suffices to solve the cases $k=7$ in (6) or its mirror images for Theorem 3 and the cases $k=8$ in (5) or its mirror images for Theorem 2.

2. Notation and lemmas. We introduce some notation. Let

$$
R=\left\{a_{i}: 0 \leq i<k\right\}
$$

and, for a prime $q$, put 
(7)

$$
S=S(q)=\{a \in R: P(a) \leq q\}, \quad S_{1}=S_{1}(q)=\{a \in R: P(a)>q\} .
$$

Further we write

$$
T=T(q)=\left\{i: a_{i} \in S\right\}, \quad T_{1}=T_{1}(q)=\left\{i: a_{i} \in S_{1}\right\} .
$$

Then we see that

$$
|T|+\left|T_{1}\right|=k .
$$

For $a \in R$, let

$$
\begin{gathered}
\nu(a)=\left|\left\{i: a_{i}=a\right\}\right|, \\
\nu_{\mathrm{o}}(a)=\left|\left\{i: a_{i}=a, 2 \nmid x_{i}\right\}\right|, \quad \nu_{\mathrm{e}}(a)=\left|\left\{i: a_{i}=a, 2 \mid x_{i}\right\}\right| .
\end{gathered}
$$

We observe that

$$
|T|=\sum_{a \in S} \nu(a)
$$

Let

$$
\delta=\min \left(3, \operatorname{ord}_{2}(d)\right), \quad \varrho= \begin{cases}3 & \text { if } 3 \mid d, \\ 1 & \text { otherwise }\end{cases}
$$

We have

Lemma 1. For $a \in R$, let $\mathcal{K}_{a}=k / a 2^{3-\delta}, \mathcal{K}_{a}^{\prime}=k / 16 a$,

$$
f_{1}(k, a, \delta)= \begin{cases}1 & \text { if } k \leq a 2^{3-\delta}, \\ \left\lceil\mathcal{K}_{a}\right\rceil-\left\lceil\frac{\left\lceil\mathcal{K}_{a}\right\rceil}{4}\right] & \text { if } k>a 2^{3-\delta}, 3 \mid d, \\ \sum_{i=1}^{2}\left(\left\lceil\frac{\mathcal{K}_{a}}{\left.\left.3^{i}\right\rceil-\left\lceil\frac{\left\lceil\mathcal{K}_{a} / 3^{i}\right\rceil}{4}\right]\right)}\right.\right. & \text { if } k>a 2^{3-\delta}, 3 \nmid d,\end{cases}
$$

and

$$
f_{2}(k, a)= \begin{cases}1 & \text { if } k \leq 4 a, \\ \left\lceil\mathcal{K}_{a}^{\prime}\right\rceil+1 & \text { if } 4 a<k \leq 32 a, \\ \sum_{i=1}^{2}\left(\left\lceil\frac{\mathcal{K}_{a}^{\prime}}{i}\right\rceil-\left\lceil\frac{\left\lceil\mathcal{K}_{a}^{\prime} / i\right\rceil}{4}\right]\right) & \text { if } k>32 a, 3 \mid d, \\ \sum_{i=1}^{2}\left(\left\lceil\frac{\mathcal{K}_{a}^{\prime}}{3^{i}}\right\rceil-\left\lceil\frac{\left\lceil\mathcal{K}_{a}^{\prime} / 3^{i}\right\rceil}{4}\right]\right)+\sum_{i=1}^{2}\left(\left\lceil\frac{\mathcal{K}_{a}^{\prime}}{2 \cdot 3^{i}}\right\rceil-\left\lceil\frac{\left\lceil\mathcal{K}_{a}^{\prime} / 2 \cdot 3^{i}\right\rceil}{4}\right]\right) \\ \text { if } k>32 a, 3 \nmid d .\end{cases}
$$

Then we have

$$
\nu_{\mathrm{o}}(a) \leq f_{1}(k, a, \delta), \quad \nu_{\mathrm{e}}(a) \leq f_{2}(k, a),
$$


and

$$
\nu(a) \leq F(k, a, \delta):= \begin{cases}1 & \text { if } k \leq a, \\ f_{1}(k, a, \delta) & \text { if } k>a \text { and } d \text { even } \\ f_{1}(k, a, 0)+f_{2}(k, a) & \text { if } k>a \text { and } d \text { odd. }\end{cases}
$$

Proof. Let $I_{1}=\left\{i: a_{i}=a, x_{i}\right.$ odd $\}, I_{2}=\left\{i: a_{i}=a, 2 \| x_{i}\right\}$ and $I_{3}=\left\{i: a_{i}=a, 4 \mid x_{i}\right\}$. Further, for $l=1,2,3$, let

$$
I_{l 1}:=\left\{i \in I_{l}: 3 \nmid x_{i}\right\}, \quad I_{l 2}:=\left\{i \in I_{l}: 3 \mid x_{i}\right\} .
$$

Let $\tau:=\tau(l, m)$ be defined by $\tau / a=2^{3-\delta} \cdot 3 \varrho^{-1}, 2^{3-\delta} \cdot 9,32 \cdot 3 \varrho^{-1}, 32 \cdot 9$, $16 \cdot 3 \varrho^{-1}, 16 \cdot 9$ for $(l, m)=(1,1),(1,2),(2,1),(2,2),(3,1),(3,2)$, respectively. Since $x_{i}^{2} \equiv 1(\bmod 8)$ for $i \in I_{1},\left(x_{i} / 2\right)^{2} \equiv 1(\bmod 8)$ for $i \in I_{2}, 16 \mid x_{i}^{2}$ for $i \in I_{3}$ and $x_{i}^{2} \equiv 1(\bmod 3)$ for $i \in I_{l 1}, 9 \mid x_{i}^{2}$ for $i \in I_{l 2}$ for $l=1,2,3$, we see from $(i-j) d=a\left(x_{i}^{2}-x_{j}^{2}\right)$ that $\tau \mid i-j$ for $i, j \in I_{l m}$. Since $a \mid i-j$ whenever $a_{i}=a_{j}$, we get $\nu(a)=1$ for $k \leq a$. Thus we suppose that $k>a$. We have $\nu(a)=\nu_{\mathrm{o}}(a)+\nu_{\mathrm{e}}(a)$. It suffices to show $\nu_{\mathrm{o}}(a) \leq f_{1}(k, a, \delta)$ and $\nu_{\mathrm{e}}(a) \leq f_{2}(k, a)$ since $\nu_{\mathrm{e}}(a)=0$ for $d$ even. We observe that $\nu_{\mathrm{O}}(a)=\left|I_{1}\right|$ and $\nu_{\mathrm{e}}(a)=\left|I_{2}\right|+\left|I_{3}\right|$. Since $a 2^{3-\delta} \mid i-j$ whenever $i, j \in I_{1}$, we get $\left|I_{1}\right| \leq 1$ if $k \leq a 2^{3-\delta}$. Thus we suppose $k>a 2^{3-\delta}$ for proving $\left|I_{1}\right| \leq f_{1}(k, a, \delta)$. Further from $4 a \mid i-j$ for $i, j \in I_{2} \cup I_{3}, 32 a \mid i-j$ for $i, j \in I_{2}$ and $16 a \mid i-j$ for $i, j \in I_{3}$, we get $\left|I_{2}\right|+\left|I_{3}\right| \leq f_{2}(k, a)$ for $k \leq 32 a$. Hence we suppose that $k>32 a$ for showing $\left|I_{2}\right|+\left|I_{3}\right| \leq f_{2}(k, a)$.

Let $(l, m)$ have $1 \leq l \leq 3,1 \leq m \leq 2$. Let $i_{0}=\min _{i \in I_{l m}} i, N=$ $\left(n+i_{0} d\right) / a$ and $D=\tau d / a$. Then we see that $a x_{i}^{2}$ with $i \in I_{l m}$ come from the squares in the set $\left\{N, N+D, \ldots, N+\left(\left\lceil\left(k-i_{0}\right) / \tau\right\rceil-1\right) D\right\}$. Dividing this set into consecutive intervals of length 4 and using Euler's result, we see that there are at most

$$
\left\lceil\frac{k-i_{0}}{\tau}\right\rceil-\left\lceil\frac{\left\lceil\left(k-i_{0}\right) / \tau\right\rceil}{4}\right] \leq\left\lceil\frac{k}{\tau}\right\rceil-\left\lceil\frac{\lceil k / \tau\rceil}{4}\right]
$$

of them which can be squares. Hence $\left|I_{l m}\right| \leq\lceil k / \tau\rceil-[\lceil k / \tau\rceil / 4]$. Now the assertion follows from $\left|I_{l}\right|=\sum_{m=1}^{2}\left|I_{l m}\right|$ for $l=1,2,3$ since $\left|I_{l 2}\right|=0$ for $3 \mid d$.

We observe that there are $(p-1) / 2$ distinct quadratic residues and $(p-1) / 2$ distinct quadratic nonresidues modulo an odd prime $p$. The next lemma follows easily from this fact.

Lemma 2. Assume (2) holds. Let $k$ be an odd prime. Suppose that $k \nmid d$. Let

$$
T^{\prime}=\left\{i:\left(\frac{a_{i}}{k}\right)=1,0 \leq i<k\right\}, \quad T^{\prime \prime}=\left\{i:\left(\frac{a_{i}}{k}\right)=-1,0 \leq i<k\right\} .
$$


Then

$$
\left|T^{\prime}\right|=\left|T^{\prime \prime}\right|=\frac{k-1}{2} .
$$

LEMMA 3. Assume that (2) with $P(b) \leq k$ has no solution at $k=k_{1}$ with $k_{1}$ prime. Then $(2)$ with $P(b) \leq k$ has no solution at $k$ with $k_{1} \leq k<k_{2}$, where $k_{2}$ is the smallest prime larger than $k_{1}$.

Proof. Let $k_{1}$ and $k_{2}$ be consecutive primes such that $k_{1} \leq k<k_{2}$. Assume that $(2)$ does not hold at $\left(n, d, k_{1}\right)$. Suppose

$$
n(n+d) \cdots(n+(k-1) d)=b y^{2} .
$$

Using (3), we see that

$$
n(n+d) \cdots\left(n+\left(k_{1}-1\right) d\right)=b^{\prime} y^{\prime 2}
$$

with $P\left(b^{\prime}\right) \leq k_{1}$. This is not possible.

Let $q_{1}, q_{2}$ be distinct primes and

$$
\Lambda_{1}\left(q_{1}, q_{2}\right):=\left\{p \leq 97:\left(\frac{p}{q_{1}}\right) \neq\left(\frac{p}{q_{2}}\right)\right\} .
$$

We write $\Lambda\left(q_{1}, q_{2}\right)=\Lambda\left(q_{1}, q_{2}, k\right):=\left\{p \in \Lambda_{1}\left(q_{1}, q_{2}\right): p \leq k\right\}$.

LEMMA 4. We have

\begin{tabular}{cc}
\hline$\left(q_{1}, q_{2}\right)$ & $\Lambda_{1}\left(q_{1}, q_{2}\right)$ \\
\hline$(5,11)$ & $\{3,19,23,29,37,41,47,53,61,67,79,97\}$ \\
$(7,17)$ & $\{11,13,19,23,29,37,47,59,71,79,83,89\}$ \\
$(11,13)$ & $\{5,17,29,31,37,43,47,59,61,67,71,79,89,97\}$ \\
$(11,59)$ & $\{7,17,19,23,29,31,37,41,47,67,79,89,97\}$ \\
$(11,61)$ & $\{13,19,23,31,37,41,53,59,67,71,73,83,89\}$ \\
$(19,29)$ & $\{11,13,17,43,47,53,59,61,67,71,73\}$ \\
$(23,73)$ & $\{13,19,29,31,37,47,59,61,67,79,89,97\}$ \\
$(23,97)$ & $\{11,13,29,41,43,53,59,61,71,79,89\}$ \\
$(31,89)$ & $\{7,11,17,19,41,53,59,73,79\}$ \\
$(37,83)$ & $\{17,23,29,31,47,53,59,61,67,71,73\}$ \\
$(41,79)$ & $\{11,13,19,37,43,59,61,67,89,97\}$ \\
$(43,53)$ & $\{7,23,29,31,37,41,67,79,83,89\}$ \\
$(43,67)$ & $\{11,13,19,29,31,37,41,53,71,73,79,89,97\}$ \\
$(53,67)$ & $\{7,11,13,19,23,43,71,73,83,97\}$ \\
$(59,61)$ & $\{7,13,17,29,47,53,71,73,79,83,97\}$ \\
$(73,97)$ & $\{11,19,23,31,37,41,43,47,53,67,71\}$ \\
$(79,89)$ & $\{13,17,19,23,31,47,53,71,83\}$ \\
\hline
\end{tabular}


Definition. Let $\mathcal{P}$ be a set of primes and $\mathcal{I} \subseteq[0, k) \cap \mathbb{Z}$. We say that $\mathcal{I}$ is covered by $\mathcal{P}$ if, for every $j \in \mathcal{I}$, there exists $p \in \mathcal{P}$ such that $p \mid a_{j}$. Further, for $i \in \mathcal{I}$, let

$$
\mathfrak{i}(\mathcal{P})=\mid\left\{p \in \mathcal{P}: p \text { divides } a_{i}\right\} \mid .
$$

For a prime $p$ with $\operatorname{gcd}(p, d)=1$, let $i_{p}$ be the smallest $i \geq 0$ such that $p \mid n+i d$. For $\mathcal{I} \subseteq[0, k) \cap \mathbb{Z}$ and primes $p_{1}, p_{2}$ with $\operatorname{gcd}\left(p_{1} p_{2}, d\right)=1$, we write

$$
\mathcal{I}^{\prime}=\mathcal{I}\left(p_{1}, p_{2}\right)=\mathcal{I} \backslash \bigcup_{j=1}^{2}\left\{i_{p_{j}}+p_{j} i: 0 \leq i<\left\lceil k / p_{j}\right\rceil\right\} .
$$

Lemma 5. Let $\mathcal{P}_{0}$ be a set of primes. Let $p_{1}, p_{2}$ be primes such that $\operatorname{gcd}\left(p_{1} p_{2}, d\right)=1$. Let $\left(i_{1}, i_{2}\right)=\left(i_{p_{1}}, i_{p_{2}}\right), \mathcal{I} \subseteq[0, k) \cap \mathbb{Z}$ and $\mathcal{I}^{\prime}=\mathcal{I}\left(p_{1}, p_{2}\right)$ be such that $\mathfrak{i}\left(\mathcal{P}_{0} \cap \Lambda\left(p_{1}, p_{2}\right)\right)$ is even for each $i \in \mathcal{I}^{\prime}$. Define

$$
\begin{aligned}
& \mathcal{I}_{1}=\left\{i \in \mathcal{I}^{\prime}:\left(\frac{i-i_{1}}{p_{1}}\right)=\left(\frac{i-i_{2}}{p_{2}}\right)\right\}, \\
& \mathcal{I}_{2}=\left\{i \in \mathcal{I}^{\prime}:\left(\frac{i-i_{1}}{p_{1}}\right) \neq\left(\frac{i-i_{2}}{p_{2}}\right)\right\} .
\end{aligned}
$$

Let $\mathcal{P}=\Lambda\left(p_{1}, p_{2}\right) \backslash \mathcal{P}_{0}$. Let $\ell$ be the number of terms $n+i d$ with $i \in \mathcal{I}^{\prime}$ divisible by primes in $\mathcal{P}$. Then either

$$
\left|\mathcal{I}_{1}\right| \leq \ell, \quad \mathcal{I}_{1} \text { is covered by } \mathcal{P}, \quad \mathcal{I}_{2}=\left\{i \in \mathcal{I}^{\prime}: \mathfrak{i}(\mathcal{P}) \text { is even }\right\}
$$

or

$$
\left|\mathcal{I}_{2}\right| \leq \ell, \quad \mathcal{I}_{2} \text { is covered by } \mathcal{P}, \quad \mathcal{I}_{1}=\left\{i \in \mathcal{I}^{\prime}: \mathfrak{i}(\mathcal{P}) \text { is even }\right\}
$$

We observe that $\ell \leq \sum_{p \in \mathcal{P}}\lceil k / p\rceil$.

Proof. Let $i \in \mathcal{I}^{\prime}$. Let $\mathcal{U}_{0}=\left\{p: p \mid a_{i}\right\}, \mathcal{U}_{1}=\left\{p \in \mathcal{U}_{0}: p \notin \Lambda\left(p_{1}, p_{2}\right)\right\}$, $\mathcal{U}_{2}=\left\{p \in \mathcal{U}_{0}: p \in \mathcal{P}_{0} \cap \Lambda\left(p_{1}, p_{2}\right)\right\}$ and $\mathcal{U}_{3}=\left\{p \in \mathcal{U}_{0}: p \in \mathcal{P}\right\}$. Then we deduce from $a_{i}=\prod_{p \in \mathcal{U}_{0}} p$ that

$$
\begin{aligned}
\left(\frac{a_{i}}{p_{1}}\right) & =\prod_{p \in \mathcal{U}_{1}}\left(\frac{p}{p_{1}}\right) \prod_{p \in \mathcal{U}_{2}}\left(\frac{p}{p_{1}}\right) \prod_{p \in \mathcal{U}_{3}}\left(\frac{p}{p_{1}}\right)=(-1)^{\mathfrak{i}(\mathcal{P})+\left|\mathcal{U}_{2}\right|} \prod_{p \in \mathcal{U}_{0}}\left(\frac{p}{p_{2}}\right) \\
& =(-1)^{\mathfrak{i}(\mathcal{P})}\left(\frac{a_{i}}{p_{2}}\right)
\end{aligned}
$$

since $\left|\mathcal{U}_{2}\right|=\mathfrak{i}\left(\mathcal{P}_{0} \cap \Lambda\left(p_{1}, p_{2}\right)\right)$ is even. Therefore

$$
\mathcal{L}:=\left\{i \in \mathcal{I}^{\prime}:\left(\frac{a_{i}}{p_{1}}\right) \neq\left(\frac{a_{i}}{p_{2}}\right)\right\}=\left\{i \in \mathcal{I}^{\prime}: \mathfrak{i}(\mathcal{P}) \text { is odd }\right\} .
$$

In particular, $\mathcal{L}$ is covered by $\mathcal{P}$ and hence

$$
|\mathcal{L}| \leq \ell .
$$


We see that $\left(\frac{a_{i}}{p_{j}}\right)=\left(\frac{n+i d}{p_{j}}\right)=\left(\frac{i-i_{j}}{p_{j}}\right)\left(\frac{d}{p_{j}}\right)$ for $i \in \mathcal{I}^{\prime}$ and $j=1,2$. Therefore $\mathcal{L}=\mathcal{I}_{1}$ or $\mathcal{I}_{2}$ according as $\left(\frac{d}{p_{1}}\right) \neq\left(\frac{d}{p_{2}}\right)$ or $\left(\frac{d}{p_{1}}\right)=\left(\frac{d}{p_{2}}\right)$, respectively. Now the assertion of Lemma 5 follows from (12) and (13).

REMARK. Let $\mathcal{P}$ consist of one prime $p$. We observe that $p \mid n+i d$ if and only if $p \mid i-i_{p}$. Then $\mathcal{I}_{1}$ or $\mathcal{I}_{2}$ is contained in one residue class modulo $p$ and $p \nmid a_{i}$ for $i$ in the other set.

Corollary 1 . Let $p_{1}, p_{2}, i_{1}, i_{2}, \mathcal{P}_{0}, \mathcal{P}, \mathcal{I}, \mathcal{I}^{\prime}, \mathcal{I}_{1}, \mathcal{I}_{2}$ and $\ell$ be as in Lemma 5. Assume that

$$
\ell<\frac{1}{2}\left|\mathcal{I}^{\prime}\right|
$$

Then $\left|\mathcal{I}_{1}\right| \neq\left|\mathcal{I}_{2}\right|$. Let

$$
\begin{gathered}
\mathcal{M}= \begin{cases}\mathcal{I}_{1} & \text { if }\left|\mathcal{I}_{1}\right|<\left|\mathcal{I}_{2}\right|, \\
\mathcal{I}_{2} & \text { otherwise, }\end{cases} \\
\mathcal{B}= \begin{cases}\mathcal{I}_{2} & \text { if }\left|\mathcal{I}_{1}\right|<\left|\mathcal{I}_{2}\right|, \\
\mathcal{I}_{1} & \text { otherwise. }\end{cases}
\end{gathered}
$$

Then $|\mathcal{M}| \leq \ell, \mathcal{M}$ is covered by $\mathcal{P}$ and $\mathcal{B}=\left\{i \in \mathcal{I}^{\prime}: \mathfrak{i}(\mathcal{P})\right.$ is even $\}$.

Proof. We see from Lemma 5 that $\min \left(\left|\mathcal{I}_{1}\right|,\left|\mathcal{I}_{2}\right|\right) \leq \ell$ and from (14) that $\max \left(\left|\mathcal{I}_{1}\right|,\left|\mathcal{I}_{2}\right|\right) \geq \frac{1}{2}\left|\mathcal{I}^{\prime}\right|>\ell$. Now the assertion follows from Lemma 5 .

We say that $(\mathcal{M}, \mathcal{B}, \mathcal{P}, \ell)$ has Property $\mathfrak{H}$ if $|\mathcal{M}| \leq \ell, \mathcal{M}$ is covered by $\mathcal{P}$ and $\mathfrak{i}(\mathcal{P})$ is even for $i \in \mathcal{B}$.

Lemma 6. Let $k$ be a prime with $7 \leq k \leq 97$ and assume (2). For $k \geq 11$, assume that Theorem 4 is valid for all primes $k_{1}$ with $7 \leq k_{1}<k$. For $11 \leq k \leq 29$, assume that $k \nmid d$ and $k \nmid n+i d$ for $0 \leq i<k-k^{\prime}$ and $k^{\prime} \leq i<k$ where $k^{\prime}<k$ are consecutive primes. Let $\left(q_{1}, q_{2}\right)=(5,7)$ if $k=7 ;(5,11)$ if $k=11 ;(11,13)$ if $13 \leq k \leq 23 ;(19,29)$ if $29 \leq k \leq 59$; $(59,61)$ if $k=61 ;(43,67)$ if $k=67,71 ;(23,73)$ if $k=73,79 ;(37,83)$ if $k=83 ;(79,89)$ if $k=89$; and $(23,97)$ if $k=97$. Then $q_{1} \mid d$ or $q_{2} \mid d$ unless $\left(a_{0}, a_{1}, \ldots, a_{k-1}\right)$ is given by the following tuples or their mirror images.

$$
\begin{aligned}
k=7: & (2,3,1,5,6,7,2),(3,1,5,6,7,2,1),(1,5,6,7,2,1,10) ; \\
k=13: & (3,1,5,6,7,2,1,10,11,3,13,14,15),(1,5,6,7,2,1,10,11,3,13,14,15,1) ; \\
k=19: & (1,5,6,7,2,1,10,11,3,13,14,15,1,17,2,19,5,21,22) ; \\
k=23: & (5,6,7,2,1,10,11,3,13,14,15,1,17,2,19,5,21,22,23,6,1,26,3), \\
& (6,7,2,1,10,11,3,13,14,15,1,17,2,19,5,21,22,23,6,1,26,3,7) .
\end{aligned}
$$

We shall prove Lemma 6 in Section 3.

Lemma 7. Let $k$ be a prime with $29 \leq k \leq 97$ and $Q_{0}$ a prime dividing $d$. Assume (2) with $k \nmid d$ and $k \nmid n+i d$ for $0 \leq i<k-k^{\prime}$ and $k^{\prime} \leq i<k$ where $k^{\prime}<k$ are consecutive primes. Then there are primes $Q_{1}$ and $Q_{2}$ given in the following table such that either $Q_{1} \mid d$ or $Q_{2} \mid d$ : 


\begin{tabular}{ccccccc}
\hline$k$ & $Q_{0}$ & $\left(Q_{1}, Q_{2}\right)$ & & $k$ & $Q_{0}$ & $\left(Q_{1}, Q_{2}\right)$ \\
\cline { 1 - 3 } \cline { 5 - 6 } $29 \leq k \leq 59$ & 19 & $(7,17)$ & & 73,79 & 23 & $(53,67)$ \\
$31 \leq k \leq 59$ & 29 & $(7,17)$ & & 79 & 73 & $(53,67)$ \\
61 & 59 & $(11,61)$ & & 83 & 37 & $(23,73)$ \\
67,71 & 43 & $(53,67)$ & & 89 & 79 & $(23,73)$ \\
71 & 67 & $(43,53)$ & & 97 & 23 & $(73,97),(37,83)$ \\
\hline
\end{tabular}

The proofs of Lemmas 6 and 7 depend on the repeated application of Lemma 5 and Corollary 1. We shall prove Lemma 7 in Section 4. Next we shall apply Lemmas 1, 2 and 7 to prove the following result.

Lemma 8. Let $k$ be a prime with $7 \leq k \leq 97$. Assume (2) with $k \nmid d$. Further for $k \geq 29$, assume that $k \nmid n+i d$ for $0 \leq i<k-k^{\prime}$ and $k^{\prime} \leq i<k$ where $k^{\prime}<k$ are consecutive primes. Let $\left(q_{1}, q_{2}\right)$ be as in Lemma 6 . Then $q_{1} \nmid d$ and $q_{2} \nmid d$.

Section 5 contains a proof of Lemma 8. Assume that $3 \nmid d$ and $5 \nmid d$. We define some more notation. For a subset $\mathcal{J} \subseteq[0, k) \cap \mathbb{Z}$, let

$$
\begin{aligned}
& \mathcal{I}_{3}^{0}=\mathcal{I}_{3}^{0}(\mathcal{J}):=\left\{i \in \mathcal{J}: i \equiv i_{3}(\bmod 3)\right\}, \\
& \mathcal{I}_{3}^{+}=\mathcal{I}_{3}^{+}(\mathcal{J}):=\left\{i \in \mathcal{J}:\left(\frac{i-i_{3}}{3}\right)=1\right\}, \\
& \mathcal{I}_{3}^{-}=\mathcal{I}_{3}^{-}(\mathcal{J}):=\left\{i \in \mathcal{J}:\left(\frac{i-i_{3}}{3}\right)=-1\right\}
\end{aligned}
$$

and

$$
\begin{aligned}
& \mathcal{I}_{5}^{+}=\mathcal{I}_{5}^{+}(\mathcal{J}):=\left\{i \in \mathcal{J}:\left(\frac{i-i_{5}}{5}\right)=1\right\}, \\
& \mathcal{I}_{5}^{-}=\mathcal{I}_{5}^{-}(\mathcal{J}):=\left\{i \in \mathcal{J}:\left(\frac{i-i_{5}}{5}\right)=-1\right\} .
\end{aligned}
$$

Assume that $a_{i} \in\{1,2,7,14\}$ for $i \in \mathcal{I}_{3}^{+} \cup \mathcal{I}_{3}^{-}$. Then either $a_{i} \in\{1,7\}$ for $i \in$ $\mathcal{I}_{3}^{+}, a_{i} \in\{2,14\}$ for $i \in \mathcal{I}_{3}^{-}$or $a_{i} \in\{2,14\}$ for $i \in \mathcal{I}_{3}^{+}, a_{i} \in\{1,7\}$ for $i \in \mathcal{I}_{3}^{-}$. We define $\left(\mathcal{I}_{3}^{1}, \mathcal{I}_{3}^{2}\right)=\left(\mathcal{I}_{3}^{+}, \mathcal{I}_{3}^{-}\right)$in the former case and $\left(\mathcal{I}_{3}^{1}, \mathcal{I}_{3}^{2}\right)=\left(\mathcal{I}_{3}^{-}, \mathcal{I}_{3}^{+}\right)$in the latter. We observe that $i$ 's have the same parity whenever $a_{i} \in\{2,14\}$. Thus if $i$ 's have the same parity in one of $\mathcal{I}_{3}^{+}$or $\mathcal{I}_{3}^{-}$but not in both, then we see that $\left(\mathcal{I}_{3}^{1}, \mathcal{I}_{3}^{2}\right)=\left(\mathcal{I}_{3}^{+}, \mathcal{I}_{3}^{-}\right)$or $\left(\mathcal{I}_{3}^{-}, \mathcal{I}_{3}^{+}\right)$according as $i$ 's have the same parity in $\mathcal{I}_{3}^{-}$or $\mathcal{I}_{3}^{+}$, respectively. Further we write

$$
\mathcal{J}_{1}=\mathcal{I}_{3}^{1} \cap \mathcal{I}_{5}^{+}, \quad \mathcal{J}_{2}=\mathcal{I}_{3}^{1} \cap \mathcal{I}_{5}^{-}, \quad \mathcal{J}_{3}=\mathcal{I}_{3}^{2} \cap \mathcal{I}_{5}^{+}, \quad \mathcal{J}_{4}=\mathcal{I}_{3}^{2} \cap \mathcal{I}_{5}^{-}
$$

and $\mathfrak{a}_{\mu}=\left\{a_{i}: i \in \mathcal{J}_{\mu}\right\}$ for $1 \leq \mu \leq 4$. Since $\left(\frac{1}{5}\right)=\left(\frac{14}{5}\right)=1$ and $\left(\frac{2}{5}\right)=\left(\frac{7}{5}\right)$ $=-1$, we see that

$$
\left(\mathfrak{a}_{1}, \mathfrak{a}_{2}, \mathfrak{a}_{3}, \mathfrak{a}_{4}\right) \subseteq(\{1\},\{7\},\{14\},\{2\}) \quad \text { or } \quad(\{7\},\{1\},\{2\},\{14\})
$$


where $\left(\mathfrak{a}_{1}, \mathfrak{a}_{2}, \mathfrak{a}_{3}, \mathfrak{a}_{4}\right) \subseteq\left(S_{1}, S_{2}, S_{3}, S_{4}\right)$ denotes $\mathfrak{a}_{\mu} \subseteq S_{\mu}, 1 \leq \mu \leq 4$. We use $7 \mid i-i^{\prime}$ whenever $a_{i}, a_{i^{\prime}} \in\{7,14\}$ to exclude one of the above possibilities.

3. Proof of Lemma 6. Let $k^{\prime}<k$ be consecutive primes. We may suppose that if (2) holds for some $k>29$, then $k \nmid d$ and $k \nmid a_{i}$ for $0 \leq i<k-k^{\prime}$ and $k^{\prime} \leq i<k$, otherwise the assertion follows from Theorem 4 with $k$ replaced by $k^{\prime}$. Subsections 3.1 to 3.10 will be devoted to the proof of Lemma 6 . We may assume that $q_{1} \nmid d$ and $q_{2} \nmid d$, otherwise the assertion follows.

3.1. The case $k=7$. Then $5 \nmid d$. By taking the mirror images (4) of (2), there is no loss of generality in assuming that $5\left|n+i_{5} d, 7\right| n+i_{7} d$ for some pair $\left(i_{5}, i_{7}\right)$ with $0 \leq i_{5}<5,0 \leq i_{7} \leq 3$. Further we may suppose $i_{7} \geq 1$, otherwise the assertion follows from the case $k=6$. We apply Lemma 5 with $\mathcal{P}_{0}=\emptyset, p_{1}=5, p_{2}=7,\left(i_{1}, i_{2}\right)=\left(i_{5}, i_{7}\right), \mathcal{I}=[0, k) \cap \mathbb{Z}, \mathcal{P}=\Lambda(5,7)=\{2\}$ and $\ell \leq \ell_{1}=\lceil k / 2\rceil$ to conclude that either

$$
\left|\mathcal{I}_{1}\right| \leq \ell_{1}, \quad \mathcal{I}_{1} \text { is covered by } \mathcal{P}, \quad \mathcal{I}_{2}=\left\{i \in \mathcal{I}^{\prime}: \mathfrak{i}(\mathcal{P}) \text { is even }\right\},
$$

or

$$
\left|\mathcal{I}_{2}\right| \leq \ell_{1}, \quad \mathcal{I}_{2} \text { is covered by } \mathcal{P}, \quad \mathcal{I}_{1}=\left\{i \in \mathcal{I}^{\prime}: \mathfrak{i}(\mathcal{P}) \text { is even }\right\} .
$$

Let $\left(i_{5}, i_{7}\right)=(3,1)$. Then $\mathcal{I}_{1}=\{0,2,6\}$ and $\mathcal{I}_{2}=\{4,5\}$. We see that $\mathcal{I}_{1}$ is covered by $\mathcal{P}$ and hence $\mathfrak{i}(\mathcal{P})$ is even for $i \in \mathcal{I}_{2}$. Thus $2 \nmid a_{i}$ for $i \in \mathcal{I}_{2}$. Therefore $a_{4}, a_{5} \in\{1,3\}$ and $a_{0}, a_{2}, a_{6} \in\{2,6\}$. If $a_{0}=6$ or $a_{6}=6$, then $3 \nmid a_{4} a_{5}$ so that $a_{4}=a_{5}=1$. This is not possible by modulo 3 . Thus $a_{0}=a_{6}$ $=2$. Since $\left(\frac{a_{0}}{5}\right)\left(\frac{a_{2}}{5}\right)=\left(\frac{(-3 d)(-d)}{5}\right)=-1$, we get $a_{2}=6$. Hence $a_{4}=1$. Further $a_{5}=3$ since $\left(\frac{a_{5}}{5}\right)\left(\frac{a_{4}}{5}\right)=\left(\frac{(2 d)(1 d)}{5}\right)=-1$. Also $5 \mid a_{3}$ and $7 \mid a_{1}$, otherwise the assertion follows from the results of [MS03] for $k=5$ and [BBGH06] for $k=6$, respectively, stated in Section 1. In fact, $a_{1}=7, a_{3}=5$ by $\operatorname{gcd}\left(a_{1} a_{3}, 6\right)=1$. Thus $\left(a_{0}, a_{1}, a_{2}, a_{3}, a_{4}, a_{5}, a_{6}\right)=(2,7,6,5,1,3,2)$. The proofs for the other cases of $\left(i_{5}, i_{7}\right)$ are similar. We get $\left(a_{0}, \ldots, a_{6}\right)=$ $(1,5,6,7,2,1,10)$ when $\left(i_{5}, i_{7}\right)=(1,3),\left(a_{0}, \ldots, a_{6}\right)=(1,2,7,6,5,1,3)$ when $\left(i_{5}, i_{7}\right)=(4,2)$ and all the other pairs are excluded. Hence Lemma 6 with $k=7$ follows.

3.2. The case $k=11$. Then $5 \nmid d$. By taking the mirror images (4) of (2), there is no loss of generality in assuming that $5\left|n+i_{5} d, 11\right| n+i_{11} d$ for some pair $\left(i_{5}, i_{11}\right)$ with $0 \leq i_{5}<5,4 \leq i_{11} \leq 5$. We apply Lemma 5 with $\mathcal{P}_{0}=\emptyset$, $p_{1}=5, p_{2}=11,\left(i_{1}, i_{2}\right)=\left(i_{5}, i_{11}\right), \mathcal{I}=[0, k) \cap \mathbb{Z}, \mathcal{P}=\Lambda(5,11)=\{3\}$ and $\ell \leq \ell_{1}=\lceil k / 3\rceil$ to derive that either

$$
\left|\mathcal{I}_{1}\right| \leq \ell_{1}, \quad \mathcal{I}_{1} \text { is covered by } \mathcal{P}, \quad \mathcal{I}_{2}=\left\{i \in \mathcal{I}^{\prime}: \mathfrak{i}(\mathcal{P}) \text { is even }\right\},
$$

or

$\left|\mathcal{I}_{2}\right| \leq \ell_{1}, \quad \mathcal{I}_{2}$ is covered by $\mathcal{P}, \quad \mathcal{I}_{1}=\left\{i \in \mathcal{I}^{\prime}: \mathfrak{i}(\mathcal{P})\right.$ is even $\}$ 
We compute $\mathcal{I}_{1}, \mathcal{I}_{2}$ and we restrict attention to those pairs $\left(i_{5}, i_{11}\right)$ for which $\min \left(\left|\mathcal{I}_{1}\right|,\left|\mathcal{I}_{2}\right|\right) \leq \ell_{1}$ and either $\mathcal{I}_{1}$ or $\mathcal{I}_{2}$ is covered by $\mathcal{P}$. We find that $\left(i_{5}, i_{11}\right)=(0,4),(1,5)$. Let $\left(i_{5}, i_{11}\right)=(0,4)$. Then $\mathcal{I}_{1}=\{3,9\}$ is covered by $\mathcal{P}, i_{3}=0$ and $\mathfrak{i}(\mathcal{P})$ is even for $i \in \mathcal{I}_{2}=\{1,2,6,7,8\}$. Thus $3 \nmid a_{i}$ for $i \in \mathcal{I}_{2}$. Further, $p \in\{2,7\}$ whenever $p \mid a_{i}$ with $i \in \mathcal{I}_{2}$. Therefore $a_{i} \in\{1,2,7,14\}$ for $i \in \mathcal{I}_{2}$. By taking $\mathcal{J}=\mathcal{I}_{2}$, we have $\mathcal{I}_{2}=\mathcal{I}_{3}^{0} \cup \mathcal{I}_{3}^{+} \cup \mathcal{I}_{3}^{-}$and $\mathcal{I}_{2}=\mathcal{I}_{5}^{+} \cup \mathcal{I}_{5}^{-}$ with

$\mathcal{I}_{3}^{0}=\{6\}, \quad \mathcal{I}_{3}^{+}=\{1,7\}, \quad \mathcal{I}_{3}^{-}=\{2,8\}, \quad \mathcal{I}_{5}^{+}=\{1,6\}, \quad \mathcal{I}_{5}^{-}=\{2,7,8\}$.

Let $\left(\mathcal{I}_{3}^{1}, \mathcal{I}_{3}^{2}\right)=\left(\mathcal{I}_{3}^{+}, \mathcal{I}_{3}^{-}\right)$. Then

$$
\mathcal{J}_{1}=\{1\}, \quad \mathcal{J}_{2}=\{7\}, \quad \mathcal{J}_{3}=\emptyset, \quad \mathcal{J}_{4}=\{2,8\} .
$$

The possibility $\left(\mathfrak{a}_{1}, \mathfrak{a}_{2}, \mathfrak{a}_{3}, \mathfrak{a}_{4}\right) \subseteq(\{7\},\{1\},\{2\},\{14\})$ is excluded since $7 \mid$ $i-i^{\prime}$ whenever $a_{i}, a_{i^{\prime}} \in\{7,14\}$. Therefore $a_{1}=1, a_{7}=7, a_{2}=a_{8}=2$. Further, $a_{6}=1$ since $6 \in \mathcal{I}_{5}^{+}$and $a_{1}=1, a_{7}=7$. This is not possible since $1=\left(\frac{a_{6}}{7}\right)\left(\frac{a_{8}}{7}\right)=\left(\frac{(-d)(d)}{7}\right)=-1$. Let $\left(\mathcal{I}_{3}^{1}, \mathcal{I}_{3}^{2}\right)=\left(\mathcal{I}_{3}^{-}, \mathcal{I}_{3}^{+}\right)$. Then we argue as above to conclude that $a_{2}=a_{8}=1, a_{1}=2, a_{7}=14$, which is not possible since $n+2 d$ and $n+8 d$ cannot both be odd squares. The other case $\left(i_{5}, i_{11}\right)=(1,5)$ is excluded similarly.

3.3. The cases $13 \leq k \leq 23$. Then $11 \nmid d$ and $13 \nmid d$. There is no loss of generality in assuming that $11\left|n+i_{11} d, 13\right| n+i_{13} d$ for some pair $\left(i_{11}, i_{13}\right)$ with $0 \leq i_{11}<11,0 \leq i_{13} \leq(k-1) / 2$ and further $i_{13} \geq 2$ if $k=13$. We have applied Lemma 5 once in each of cases $k=7$ and $k=11$ but we apply it twice for every case $13 \leq k \leq 23$ in this subsection. Let $\mathcal{P}_{0}=\emptyset$, $p_{1}=11, p_{2}=13,\left(i_{1}, i_{2}\right)=\left(i_{11}, i_{13}\right), \mathcal{I}=[0, k) \cap \mathbb{Z}, \mathcal{P}=\mathcal{P}_{1}:=\Lambda(11,13)$ and $\ell \leq \ell_{1}$ where $\ell_{1}=3$ if $k=13$, and $\ell_{1}=\lceil k / 5\rceil+\lceil k / 17\rceil$ if $k>13$. Then $\ell_{1}<\frac{1}{2}\left|\mathcal{I}^{\prime}\right|$ since $\left|\mathcal{I}^{\prime}\right| \geq k-\lceil k / 11\rceil-\lceil k / 13\rceil$. By Corollary 1 , we derive that $\mathcal{I}^{\prime}$ is partitioned into $\mathcal{M}=: \mathcal{M}_{1}$ and $\mathcal{B}=: \mathcal{B}_{1}$ such that $\left(\mathcal{M}_{1}, \mathcal{B}_{1}, \mathcal{P}_{1}, \ell_{1}\right)$ has Property $\mathfrak{H}$. Now we restrict to all such pairs $\left(i_{11}, i_{13}\right)$ satisfying $\left|\mathcal{M}_{1}\right| \leq \ell_{1}$ and $\mathcal{M}_{1}$ is covered by $\mathcal{P}_{1}$. We check that $\left|\mathcal{M}_{1}\right|>2$. Therefore $5 \nmid d$ since $\mathcal{M}_{1}$ is covered by $\mathcal{P}_{1}$. Thus there exists $i_{5}$ with $0 \leq i_{5}<5$ such that $5 \mid n+i_{5} d$.

Now we apply Lemma 5 with $p_{1}=5, p_{2}=11$ and partition $\mathcal{B}_{1}(5,11)$ into two subsets. Let $\mathcal{P}_{0}=\Lambda(11,13) \cup\{11,13\},\left(i_{1}, i_{2}\right)=\left(i_{5}, i_{11}\right), \mathcal{I}=\mathcal{B}_{1}$, $\mathcal{P}=\mathcal{P}_{2}:=\Lambda(5,11) \subseteq\{3,19,23\}$ and $\ell \leq \ell_{2}$ where $\ell_{2}=5,6,8,11$ if $k=$ $13,17,19,23$, respectively. Hence $\mathcal{B}_{1}^{\prime}$ is partitioned into $\mathcal{I}_{1}$ and $\mathcal{I}_{2}$ satisfying either

$\left|\mathcal{I}_{1}\right| \leq \ell_{2}, \quad \mathcal{I}_{1}$ is covered by $\mathcal{P}_{2}, \quad \mathcal{I}_{2}=\left\{i \in \mathcal{I}^{\prime}: \mathfrak{i}\left(\mathcal{P}_{2}\right)\right.$ is even $\}$,

or

$$
\left|\mathcal{I}_{2}\right| \leq \ell_{2}, \quad \mathcal{I}_{2} \text { is covered by } \mathcal{P}_{2}, \quad \mathcal{I}_{1}=\left\{i \in \mathcal{I}^{\prime}: \mathfrak{i}\left(\mathcal{P}_{2}\right) \text { is even }\right\} .
$$

We compute $\mathcal{I}_{1}, \mathcal{I}_{2}$ and we restrict attention to those pairs $\left(i_{11}, i_{13}\right)$ for which 
$\min \left(\left|\mathcal{I}_{1}\right|,\left|\mathcal{I}_{2}\right|\right) \leq \ell_{2}$ and either $\mathcal{I}_{1}$ or $\mathcal{I}_{2}$ is covered by $\mathcal{P}_{2}$. We find that $\left(i_{11}, i_{13}\right)=(4,2),(5,3)$ if $k=13 ;(0,0),(5,3)$ if $k=17 ;(0,0),(0,9),(7,5)$, $(7,9),(8,6),(9,7),(10,8)$ if $k=19$; and $(0,0),(0,9),(1,10),(2,11),(4,0)$, $(5,1),(5,7),(6,2),(6,8),(7,9),(8,10),(9,11)$ if $k=23$.

Let $\left(i_{11}, i_{13}\right)$ be such a pair. We write $M$ for the one of $\mathcal{I}_{1}$ or $\mathcal{I}_{2}$ which is covered by $\mathcal{P}_{2}$ and $B$ for the other. For $i \in \mathcal{B}_{1}^{\prime}$, we see that $p \nmid a_{i}$ whenever $p \in \mathcal{P}_{0}$ since $17 \mid a_{i}$ implies $5 \mid a_{i}$. Therefore

$$
\mathfrak{i}\left(\mathcal{P}_{2}\right) \text { is even for } i \in B \text { and } p \nmid a_{i} \text { for } i \in B \text { whenever } p \in \mathcal{P}_{0} \text {, }
$$

since $B \subseteq \mathcal{B}_{1}^{\prime}$. Further we check that $|M|>1$ if $k \neq 23$ and $>3$ if $k=23$, implying $3 \nmid d$.

By taking $\mathcal{J}=B$, we get $B=\mathcal{I}_{3}^{0} \cup \mathcal{I}_{3}^{+} \cup \mathcal{I}_{3}^{-}$and $B=\mathcal{I}_{5}^{+} \cup \mathcal{I}_{5}^{-}$. Then $p \in\{2,7\}$ whenever $p \mid a_{i}$ with $i \in \mathcal{I}_{3}^{+} \cup \mathcal{I}_{3}^{-}$by (18). By computing $\mathcal{I}_{3}^{+}, \mathcal{I}_{3}^{-}$, we find that $i$ 's have the same parity in exactly one of $\mathcal{I}_{3}^{+}, \mathcal{I}_{3}^{-}$. Therefore we deduce from (17) that

$$
\left(\mathfrak{a}_{1}, \mathfrak{a}_{2}, \mathfrak{a}_{3}, \mathfrak{a}_{4}\right) \subseteq(\{1\},\{7\},\{14\},\{2\}) \text { or }(\{7\},\{1\},\{2\},\{14\}) .
$$

Let $k=13$ and $\left(i_{11}, i_{13}\right)=(4,2)$. Then we have $\mathcal{M}_{1}=\{0,5,10\}, i_{5}=0$, $M=\{3,9,12\}$ and $B=\{1,6,7,8,11\}$ since the latter set is not covered by $\mathcal{P}_{2}=\{3\}$. Further $i_{3}=0, \mathcal{I}_{3}^{0}=\{6\}, \mathcal{I}_{3}^{1}=\mathcal{I}_{3}^{-}=\{8,11\}, \mathcal{I}_{3}^{2}=\mathcal{I}_{3}^{+}=\{1,7\}$, $\mathcal{I}_{5}^{+}=\{1,6,11\}, \mathcal{I}_{5}^{-}=\{7,8\}, \mathcal{J}_{1}=\{11\}, \mathcal{J}_{2}=\{8\}, \mathcal{J}_{3}=\{1\}, \mathcal{J}_{4}=\{7\}$. Hence $a_{11}=1, a_{8}=7, a_{1}=14, a_{7}=2$ or $a_{11}=7, a_{8}=1, a_{1}=2, a_{7}=14$. The second possibility is excluded since $a_{11}=7, a_{7}=14$ is not possible. Further, from (18) we get $a_{6}=1$ since $2 \nmid a_{6}$ and $7 \nmid a_{6}$. Since $13 \mid n+2 d$ and $7 \mid n+d$, we get $\left(\frac{i-2}{13}\right)=\left(\frac{a_{i} a_{6}}{13}\right)=\left(\frac{a_{i}}{13}\right)$ and $-\left(\frac{i-1}{7}\right)=\left(\frac{a_{i} a_{6}}{7}\right)=\left(\frac{a_{i}}{7}\right)$. We observe that $13|n+2 d, 11| n+4 d, 7|n+d, 5| n, 3|n, 2| n+d, 5 \mid a_{i}$ for $i \in \mathcal{M}$ and $3 \mid a_{i}$ for $i \in \mathcal{M}_{1}$. Now we see that $a_{0} \in\{5,15\}$ and $a_{0}=5$ is excluded since $\left(\frac{5}{7}\right) \neq-\left(\frac{-1}{7}\right)$. Thus $a_{0}=15$. Next $a_{1}=14, a_{2}=13$ and $a_{3}=3$. Also $a_{4} \in\{1,11\}$ and $a_{4} \neq 1$ since $\left(\frac{a_{4}}{13}\right)=\left(\frac{2}{13}\right)=-1$. Similarly we derive that $a_{5}=10, a_{6}=1, a_{7}=2, a_{8}=7, a_{9}=6, a_{10}=5, a_{11}=1$ and $a_{12}=3$. Thus $\left(a_{0}, a_{1}, \ldots, a_{12}\right)=(15,14, \ldots, 6,5,1,3)$. The other case $\left(i_{11}, i_{13}\right)=(5,3)$ is similar and we get $\left(a_{0}, a_{1}, \ldots, a_{12}\right)=(1,15,14, \ldots, 5,1)$.

Let $k=17$ and $\left(i_{11}, i_{13}\right)=(0,0)$. Then we have $\mathcal{M}_{1}=\{5,10,15\}$ and $i_{5}=0$. We see from the assumption of Lemma 6 with $k=17, k^{\prime}=13$ that $4 \leq i_{17}<13$. Hence, from $i_{17} \in \bigcup_{p=5,11,13}\left\{i_{p}+p j: 0 \leq j<\lceil k / p\rceil\right\}$, we get $i_{17} \in\{5,10,11\}$. Further $M=\{3,6,12\}, B=\{1,2,4,7,8,9,14,16\}$, $i_{3}=0, \mathcal{I}_{3}^{0}=\{9\}, \mathcal{I}_{3}^{1}=\{1,4,7,16\}, \mathcal{I}_{3}^{2}=\{2,8,14\}, \mathcal{I}_{5}^{+}=\{1,4,9,14,16\}$, $\mathcal{I}_{5}^{-}=\{2,7,8\}, \mathcal{J}_{1}=\{1,4,16\}, \mathcal{J}_{2}=\{7\}, \mathcal{J}_{3}=\{14\}$ and $\mathcal{J}_{4}=\{2,8\}$. Therefore $a_{1}=a_{4}=a_{16}=1, a_{7}=7, a_{14}=14, a_{2}=a_{8}=2$. Thus $a_{9}=1$ by (18) and $2 \nmid a_{9}, 7 \nmid a_{9}$. Now we see by the Legendre symbol mod 17 that $a_{1}=a_{4}=a_{9}=a_{16}=1$ is not possible. The case $\left(i_{11}, i_{13}\right)=(5,3)$ is excluded similarly. 
Let $k=19$ and $\left(i_{11}, i_{13}\right)=(0,0)$. Then we have $\mathcal{M}_{1}=\{5,10,15,17\}$, $i_{5}=0, i_{17}=0, M=\{3,6,12\}, B=\{1,2,4,7,8,9,14,16,18\}$ and $i_{3}=0$. We see from $i_{19} \in \bigcup_{p=3,5,11,13,17}\left\{i_{p}+p j: 0 \leq j<\lceil k / p\rceil\right\}$ and $2 \leq$ $i_{19}<17$ that $i_{19} \in\{3,5,6,9,10,11,12,13,15\}$. Further, $\mathcal{I}_{3}^{0}=\{9,18\}$, $\mathcal{I}_{3}^{1}=\{1,4,7,16\}, \mathcal{I}_{3}^{2}=\{2,8,14\}, \mathcal{I}_{5}^{+}=\{1,4,9,14,16\}, \mathcal{I}_{5}^{-}=\{2,7,8,18\}$, $\mathcal{J}_{1}=\{1,4,16\}, \mathcal{J}_{2}=\{7\}, \mathcal{J}_{3}=\{14\}$ and $\mathcal{J}_{4}=\{2,8\}$. Therefore $a_{1}=a_{4}=$ $a_{16}=1$, which is not possible by $\bmod 19$. The case $\left(i_{11}, i_{13}\right)=(7,5)$ is excluded similarly. Let $\left(i_{11}, i_{13}\right)=(0,9)$. Then $\mathcal{M}_{1}=\{2,5,7,12,17\}, i_{5}=2$, $i_{17}=5, M=\{1,3,10,16\}, B=\{4,6,8,13,14,15,18\}, i_{3}=1$ and $i_{19}=3$. We now consider $(n+6 d)(n+7 d) \cdots(n+18 d)=b^{\prime} y^{\prime 2}$. Then $P\left(b^{\prime}\right) \leq 13$. By the case $k=13$, we get $\left(a_{6}, a_{7}, \ldots, a_{18}\right)=(1,15,14, \ldots, 6,5,1)$ since $5 \mid a_{7}$ and $3 \mid a_{16}$. From $19 \mid n+3 d$, we get $\left(\frac{a_{i}}{19}\right)=\left(\frac{a_{i} a_{6}}{19}\right)=-\left(\frac{i-3}{19}\right)$ which together with $13|n+9 d, 11| n, 7|n+d, 2| n, 5\left|a_{2}, 17\right| a_{5}, 3 \mid a_{1}$ implies $a_{0} \in\{2,22\}$, $a_{1} \in\{3,21\}, a_{2}=5, a_{3}=19, a_{4}=2$ and $a_{5}=17$. Now from $\left(\frac{a_{i}}{17}\right)=\left(\frac{a_{i} a_{6}}{17}\right)=$ $\left(\frac{i-5}{17}\right)$, we get $a_{0}=22, a_{1}=21$. Thus $\left(a_{0}, a_{1}, \ldots, a_{18}\right)=(22,21, \ldots, 6,5,1)$. The case $\left(i_{11}, i_{13}\right)=(7,9)$ is similar and we get $\left(a_{0}, a_{1}, \ldots, a_{18}\right)=(1,5,6$, $\ldots, 21,22)$. For the pair $\left(i_{11}, i_{13}\right)=(10,8)$, we similarly get $\left(a_{0}, a_{1}, \ldots, a_{18}\right)$ $=(21,5, \ldots, 6,5,1,3)$. This is excluded by considering $(n+3 d)(n+6 d)$ $\cdots(n+18 d)$ and $k=6$. For the pairs $\left(i_{11}, i_{13}\right)=(8,6),(9,7)$, we get $i_{19}=0,1$, respectively, which is not possible since $i_{19} \geq 2$ by the assumption of the lemma.

Let $k=23$ and $\left(i_{11}, i_{13}\right)=(0,0)$. Then $\mathcal{M}_{1}=\{5,10,15,17,20\}, i_{5}=0$, $i_{17}=0, M=\{3,6,12,19,21\}, B=\{1,2,4,7,8,9,14,16,18\}, i_{3}=0$ and $i_{19}=0$ since $23 \nmid a_{19}$. We have $i_{23} \in\{5,6,9,10,11,12,13,15,17,18\}$ since $4 \leq i_{23}<19$. Here we observe that $23 \nmid a_{19}$ and $4 \leq i_{23}<19$ in view of our assumption that $k \nmid a_{i}$ for $0 \leq i<k-k^{\prime}$ and $k^{\prime} \leq i<k$ with $k=23$, $k^{\prime}=19$. Further, $\mathcal{I}_{3}^{0}=\{9,18\}, \mathcal{I}_{3}^{1}=\{1,4,7,16\}, \overline{\mathcal{I}_{3}^{2}}=\{2,8,14\}, \mathcal{I}_{5}^{+}=$ $\{1,4,9,14,16\}, \mathcal{I}_{5}^{-}=\{2,7,8,18\}, \mathcal{J}_{1}=\{1,4,16\}, \mathcal{J}_{2}=\{7\}, \mathcal{J}_{3}=\{14\}$ and $\mathcal{J}_{4}=\{2,8\}$. Therefore $a_{1}=a_{4}=a_{16}=1, a_{7}=7, a_{14}=14, a_{2}=a_{8}=2$. This is not possible since $\left(\frac{a_{1}}{23}\right)=\left(\frac{a_{4}}{23}\right)=\left(\frac{a_{16}}{23}\right)=\left(\frac{a_{2}}{23}\right)=\left(\frac{a_{8}}{23}\right)=1$. The cases $\left(i_{11}, i_{13}\right)=(0,9),(1,10),(2,11),(4,0),(7,9),(8,10),(9,11)$ are excluded similarly. Let $\left(i_{11}, i_{13}\right)=(5,1)$. Then $\mathcal{M}_{1}=\{7,10,12,17,22\}, i_{5}=2, i_{17}=10$, $M=\{0,3,4,6,8,15,21\}, B=\{9,11,13,18,19,20\}$ and $i_{3}=0$. This implies either $23\left|a_{4}, 19\right| a_{8}$ or $23\left|a_{8}, 19\right| a_{4}$. Further, $\mathcal{I}_{3}^{0}=\{9,18\}, \mathcal{I}_{3}^{1}=\{11,20\}$, $\mathcal{I}_{3}^{2}=\{13,19\}, \mathcal{I}_{5}^{+}=\{11,13,18\}, \mathcal{I}_{5}^{-}=\{9,19,20\}, \mathcal{J}_{1}=\{11\}, \mathcal{J}_{2}=\{20\}$, $\mathcal{J}_{3}=\{13\}$ and $\mathcal{J}_{4}=\{19\}$. Therefore $a_{11}=1, a_{20}=7, a_{13}=14, a_{19}=2$. Further, from (18) we get $a_{9} \in\{1,2\}, a_{18}=1$ since $7 \nmid a_{9} a_{18}, 2 \nmid a_{18}$. However, $a_{9}=2$ as $9 \in \mathcal{I}_{5}^{-}, 18 \in \mathcal{I}_{5}^{+}$. Since $\left(\frac{a_{11}}{23}\right)=\left(\frac{a_{18}}{23}\right)=1$, we see that $23 \mid a_{4}$, $19 \mid a_{8}$. By using $\left(\frac{a_{i}}{p}\right)=\left(\frac{a_{i} a_{11}}{p}\right)=\left(\frac{\left(i-i_{p}\right)\left(11-i_{p}\right)}{p}\right)$, we get $\left(\frac{a_{i}}{23}\right)=-\left(\frac{i-4}{23}\right)$, $\left(\frac{a_{i}}{11}\right)=-\left(\frac{i-5}{11}\right),\left(\frac{a_{i}}{7}\right)=-\left(\frac{i-6}{7}\right)$ and $\left(\frac{a_{i}}{5}\right)=\left(\frac{i-2}{5}\right)$. Now from $23\left|a_{4}, 19\right| a_{8}$, $17\left|a_{10}, 13\right| n+d, 11|n+5 d, 7| n+6 d, 5|n+2 d, 3| n, 2 \mid n+d, \mathcal{M}_{1}$ being 
covered by $\{5,17\}$, and $M$ by $\{3,19,23\}$, we derive that $\left(a_{0}, a_{1}, \ldots, a_{22}\right)=$ $(3,26, \ldots, 6,5)$. The cases $\left(i_{11}, i_{13}\right)=(5,7),(6,2),(6,8)$ are similar and we get $\left(a_{0}, a_{1}, \ldots, a_{22}\right)=(6,7, \ldots, 3,7),(7,3, \ldots, 7,6),(5,6,7, \ldots, 3)$, respectively.

3.4. Introductory remarks on the cases $k \geq 29$. Assume $q_{1} \nmid d$ and $q_{2} \nmid d$. Then, by taking the mirror image (4) of (2), there is no loss of generality in assuming that $q_{1}\left|n+i_{q_{1}} d, q_{2}\right| n+i_{q_{2}} d$ for some pair $\left(i_{q_{1}}, i_{q_{2}}\right)$ with $0 \leq$ $i_{q_{1}}<q_{1}, 0 \leq i_{q_{2}} \leq(k-1) / 2$ and further $i_{q_{2}} \geq k-k^{\prime}$ if $q_{2}=k$. For $k=61$, by taking $(n+8 d) \cdots(n+60 d)$ and $k=53$, we may assume that $\max \left(i_{59}, i_{61}\right) \geq 8$ if $i_{59} \geq 2$. Let $\mathcal{P}_{0}=\emptyset, p_{1}=q_{1}, p_{2}=q_{2},\left(i_{1}, i_{2}\right)=\left(i_{q_{1}}, i_{q_{2}}\right)$, $\mathcal{I}=[0, k) \cap \mathbb{Z}, \mathcal{P}=\mathcal{P}_{1}:=\Lambda\left(q_{1}, q_{2}\right)$ and $\ell \leq \ell_{1}=\sum_{p \in \mathcal{P}_{1}}\lceil k / p]$. We check that $\ell_{1}<\frac{1}{2}\left|\mathcal{I}^{\prime}\right|$ since $\left|\mathcal{I}^{\prime}\right| \geq k-\left\lceil k / q_{1}\right\rceil-\left\lceil k / q_{2}\right\rceil$. By Corollary 1 , we get $\mathcal{M}=: \mathcal{M}_{1}$ and $\mathcal{B}=: \mathcal{B}_{1}$ with $\left(\mathcal{M}_{1}, \mathcal{B}_{1}, \mathcal{P}_{1}, \ell_{1}\right)$ having Property $\mathfrak{H}$. We now restrict to all such pairs $\left(i_{q_{1}}, i_{q_{2}}\right)$ for which $\left|\mathcal{M}_{1}\right| \leq \ell_{1}$ and $\mathcal{M}_{1}$ is covered by $\mathcal{P}_{1}$. We find that there is no such pair $\left(i_{q_{1}}, i_{q_{2}}\right)$ when $k=97$.

3.5. The cases $29 \leq k \leq 59$. As stated in Lemma 6 , we have $q_{1}=19$, $q_{2}=29$ and $\mathcal{P}_{1}=\Lambda(19,29) \subseteq\{11,13,17,43,47,53,59\}$. Then the pairs $\left(i_{q_{1}}, i_{q_{2}}\right)$ are given by

$$
\begin{aligned}
k=29: & (0,9),(1,10),(2,11),(3,12),(4,13),(15,5),(16,6),(17,7),(18,8) ; \\
k=31: & (0,0),(0,9),(1,10),(2,11),(3,12),(4,13),(11,1) \\
& (12,2),(13,3),(14,4),(15,5),(16,6),(17,7),(18,8) ; \\
k=37: & (0,0),(0,9),(1,10),(2,11),(3,12),(4,13),(17,7),(18,8) ; \\
k=41: & (0,0),(2,11),(3,12),(4,13) ; \\
k=43: & (0,0),(1,1),(3,12),(4,13),(5,14),(6,15),(7,16),(8,17) ; \\
k=47: & (0,0),(1,1),(7,16),(8,17),(9,18),(10,19),(11,20), \\
& (12,21),(13,22),(13,23),(14,23) ; \\
k=53: & (0,0),(1,0),(1,1),(13,22),(13,23),(14,23),(14,24), \\
& (15,24),(15,25),(16,25),(16,26),(17,26) ; \\
k=59: & (0,0),(0,28),(1,0),(1,1),(2,1),(3,2),(17,27),(18,28) .
\end{aligned}
$$

Let $k=31$ and $\left(i_{19}, i_{29}\right)=(0,9)$. We see that $\mathcal{P}_{1}=\{11,13,17\}, \mathcal{M}_{1}=$ $\{4,5,12,16,21,25,27\}$ and $\mathcal{B}_{1}=\{1,2,3,6,7,8,10,11,13,14,15,17,18,20$, $22,23,24,26,28,29,30\}$. Since $\mathcal{M}_{1}$ is covered by $\mathcal{P}_{1}$, we find that 11 divides $a_{5}, a_{16}, a_{27} ; 13$ divides $a_{12}, a_{25} ;$ and 17 divides $a_{4}, a_{21}$. Hence $i_{11}=5$, $i_{13}=12, i_{17}=4$. We see that $\operatorname{gcd}\left(11 \cdot 13 \cdot 17, a_{i}\right)=1$ for $i \in \mathcal{B}_{1}$. Now we take $\mathcal{P}_{0}=\mathcal{P}_{1} \cup\{19,29\}, p_{1}=11, p_{2}=13,\left(i_{1}, i_{2}\right):=\left(i_{11}, i_{13}\right)=(5,12), \mathcal{I}=\mathcal{B}_{1}$, $\mathcal{P}=\mathcal{P}_{2}:=\Lambda(11,13) \backslash \mathcal{P}_{0}=\{5,31\}$ and $\ell \leq \ell_{2}=\sum_{p \in \mathcal{P}_{2}}\lceil k / p\rceil=8$. Thus $\left|\mathcal{I}^{\prime}\right|=\left|\mathcal{B}_{1}\right|=21>2 \ell_{2}$. Then the conditions of Corollary 1 are satisfied and we have $\mathcal{M}=: \mathcal{M}_{2}, \mathcal{B}=: \mathcal{B}_{2}$ such that $\left(\mathcal{M}_{2}, \mathcal{B}_{2}, \mathcal{P}_{2}, \ell_{2}\right)$ has Property $\mathfrak{H}$. We 
get $\mathcal{M}_{2}=\{1,3,7,8,18,23,28\}$. This is not possible since $\mathcal{M}_{2}$ is not covered by $\mathcal{P}_{2}$. Further, the following pairs $\left(i_{19}, i_{29}\right)$ are excluded similarly:

$$
\begin{aligned}
& k=29:(0,9),(1,10),(2,11),(3,12),(4,13),(15,5),(16,6),(17,7),(18,8) ; \\
& k=31:(1,10),(2,11),(3,12),(4,13),(18,8) .
\end{aligned}
$$

Thus $k>29$.

Let $k=59$ and $\left(i_{19}, i_{29}\right)=(0,0)$. Then we see that $\mathcal{P}_{1}=\{11,13,17$, $43,47,53,59\}, \mathcal{M}_{1}=\{11,13,17,22,26,33,34,39,43,44,47,51,52,53,55\}$, $\mathcal{B}_{1}=\{1,2,3,4,5,6,7,8,9,10,12,14,15,16,18,20,21,23,24,25,27,28,30$, $31,32,35,36,37,40,41,42,45,46,48,49,50,54,56\}, i_{11}=i_{13}=i_{17}=0$, $\{43,47,53\}$ is covered by $\{43,47,53,59\}=: \mathcal{P}_{1}^{\prime}$. Let $p \mid a_{i}$ for $i \in \mathcal{B}_{1}$ and $p \in \mathcal{P}_{1}$. Then we show that $i \in\{4,6,10\}$. Let $59 \mid a_{43}$. Then $\{47,53\}$ is covered by $\{43,47,53\}$. Let $43 \mid a_{47}$. If $43 \mid a_{i}$ with $i \in \mathcal{B}_{1}$, then $i=4$ and $43 p \mid a_{4}$ with $p \in\{47,53\}$ since $\mathfrak{i}\left(\mathcal{P}_{1}\right)$ is even. This implies either $53\left|a_{53}, 43 \cdot 47\right| a_{4}$ or $47\left|a_{53}, 43 \cdot 53\right| a_{4}$. Similarly we get $i \in\{4,6,10\}$ by considering all the cases $59\left|a_{43}, 59\right| a_{47}$ and $59 \nmid a_{43} a_{47} a_{53}$. We observe that $59 \nmid a_{53}$ since $6 \leq i_{59}<53$. Hence we conclude that $p \nmid a_{i}$ for $i \in \mathcal{B}_{1} \backslash\{4,6,10\}$ and $p \in \mathcal{P}_{1}^{\prime}$. Further we observe that

$$
i_{59} \in \mathcal{M}_{1} \cup\{19,29,38\} \cup\{6,10\} .
$$

Now we take $\mathcal{P}_{0}=\mathcal{P}_{1} \cup\{19,29\}, p_{1}=11, p_{2}=13,\left(i_{1}, i_{2}\right):=(0,0)$, $\mathcal{I}=\mathcal{B}_{1} \backslash\{4,6,10\}, \mathcal{P}=\mathcal{P}_{2}:=\Lambda(11,13) \backslash \mathcal{P}_{0}=\{5,31,37\}$ and $\ell \leq \ell_{2}=$ $\sum_{p \in \mathcal{P}_{2}}\lceil k / p\rceil=16$. Thus $\left|\mathcal{I}^{\prime}\right|=\left|\mathcal{B}_{1}\right|-2>2 \ell_{2}$. Then the conditions of Corollary 1 are satisfied and we have $\mathcal{M}=: \mathcal{M}_{2}, \mathcal{B}=: \mathcal{B}_{2}$ with $\left(\mathcal{M}_{2}, \mathcal{B}_{2}, \mathcal{P}_{2}, \ell_{2}\right)$ having Property $\mathfrak{H}$. We get $\mathcal{M}_{2}=\{5,15,20,30,31,35,37,40,45\}, \mathcal{B}_{2}=$ $\{1,2,3,7,8,9,12,14,16,18,21,23,24,25,27,28,32,36,41,42,46,48$, $49,50,54,56\}, i_{5}=0$, and $31\left|a_{31}, 37\right| a_{37}$ or $31\left|a_{37}, 37\right| a_{31}$. Now we take $\mathcal{P}_{0}=\mathcal{P}_{1} \cup \mathcal{P}_{2} \cup\{19,29\}, p_{1}=5, p_{2}=11,\left(i_{1}, i_{2}\right):=(0,0), \mathcal{I}=\mathcal{B}_{2}, \mathcal{P}=\mathcal{P}_{3}:=$ $\Lambda(5,11) \backslash \mathcal{P}_{0}=\{3,23,41\}$ and $\ell \leq \ell_{3}=\sum_{p \in \mathcal{P}_{3}}\lceil k / p\rceil$. Then by Lemma 5 , we see that $M=\{3,6,12,21,23,24,27,41,42,46,48,54\}$ is covered by $\mathcal{P}_{3}$ and $\mathfrak{i}\left(\mathcal{P}_{3}\right)$ is even for $i \in B=\{1,2,7,8,9,14,16,18,28,32,36,49,56\}$. Thus $i_{3}=i_{23}=i_{41}=0$ and $p \in\{2,7\}$ whenever $p \mid a_{i}$ with $i \in B$. Putting $\mathcal{J}=B$, we have $B=\mathcal{I}_{3}^{0} \cup \mathcal{I}_{3}^{1} \cup \mathcal{I}_{3}^{2}$ and $B=\mathcal{I}_{5}^{+} \cup \mathcal{I}_{5}^{-}$with

$$
\mathcal{I}_{3}^{0}=\{9,18,36\}, \quad \mathcal{I}_{3}^{1}=\{1,7,16,28,49\}, \quad \mathcal{I}_{3}^{2}=\{2,8,14,32,56\}
$$

and

$$
\mathcal{I}_{5}^{+}=\{1,9,14,16,36,49,56\}, \quad \mathcal{I}_{5}^{-}=\{2,7,8,18,28,32\}
$$

so that

$$
\mathcal{J}_{1}=\{1,16,49\}, \quad \mathcal{J}_{2}=\{7,28\}, \quad \mathcal{J}_{3}=\{14,56\}, \quad \mathcal{J}_{4}=\{2,8,32\} .
$$

Hence $\left(\mathfrak{a}_{1}, \mathfrak{a}_{2}, \mathfrak{a}_{3}, \mathfrak{a}_{4}\right) \subseteq(\{1\},\{7\},\{14\},\{2\})$ by (17). Thus $a_{1}=a_{16}=a_{49}$ $=1, a_{7}=a_{28}=7, a_{14}=a_{56}=14, a_{2}=a_{8}=a_{32}=2$. Further, we get 
$a_{9}=a_{36}=1$ and $a_{18}=2$ since $9,36 \in \mathcal{I}_{5}^{+}$and $18 \in \mathcal{I}_{5}^{-}$. Since

$$
\left(\frac{a_{i}}{59}\right)=1 \quad \text { for } a_{i} \in\{1,7\}
$$

we see that $\left(\frac{a_{i}}{59}\right)=1$ for $i \in\{1,7,9,16,28,36,49\}$, which is not possible by (19).

Let $k=41$ and $\left(i_{19}, i_{29}\right)=(2,11)$. Then we see that $\mathcal{P}_{1}=\{11,13,17\}$, $\mathcal{M}_{1}=\{1,6,7,14,18,23,27,29\}, \mathcal{B}_{1}=\{0,3,4,5,8,9,10,12,13,15,16,17,19$, $20,22,24,25,26,28,30,31,32,33,34,35,36,37,38,39\}, i_{11}=7, i_{13}=1$, $i_{17}=6$. Further $\operatorname{gcd}\left(a_{i}, 11 \cdot 13 \cdot 17\right)=1$ for $i \in \mathcal{B}_{1}$. Now we take $\mathcal{P}_{0}=$ $\mathcal{P}_{1} \cup\{19,29\}, p_{1}=11, p_{2}=13,\left(i_{1}, i_{2}\right):=(7,1), \mathcal{I}=\mathcal{B}_{1}, \mathcal{P}=\mathcal{P}_{2}:=$ $\Lambda(11,13) \backslash \mathcal{P}_{0}=\{5,31,37\}$ and $\ell \leq \ell_{2}=\sum_{p \in \mathcal{P}_{2}}\lceil k / p\rceil=13$. Then $\left|\mathcal{I}^{\prime}\right|=$ $\left|\mathcal{B}_{1}\right|>2 \ell_{2}$. Thus the conditions of Corollary 1 are satisfied and we get $\mathcal{M}=$ : $\mathcal{M}_{2}$ and $\mathcal{B}=: \mathcal{B}_{2}$ such that $\left(\mathcal{M}_{2}, \mathcal{B}_{2}, \mathcal{P}_{2}, \ell_{2}\right)$ has Property $\mathfrak{H}$. We have $\mathcal{M}_{2}=$ $\{0,3,5,9,10,20,25,30,35\}, \mathcal{B}_{2}=\{4,8,12,13,15,16,17,19,22,24,26,28,31$, $32,33,34,36,37,38,39\}, i_{5}=0$. Further $31 \cdot 37 \mid a_{3} a_{9}, 31 \nmid a_{34}$. We take $\mathcal{P}_{0}=$ $\mathcal{P}_{1} \cup \mathcal{P}_{2} \cup\{19,29\}, p_{1}=5, p_{2}=11,\left(i_{1}, i_{2}\right):=(0,7), \mathcal{I}=\mathcal{B}_{2}, \mathcal{P}=$ $\mathcal{P}_{3}:=\Lambda(5,11) \backslash \mathcal{P}_{0}=\{3,23,41\}, \ell \leq \sum_{p \in \mathcal{P}_{3}}\lceil k / p\rceil$ and apply Lemma 5 to see that $M=\{13,16,17,19,28,34,37\}$ is covered by $\mathcal{P}_{3}, i_{3}=1, \mathfrak{i}\left(\mathcal{P}_{3}\right)$ is even for $i \in B=\{4,8,12,22,24,26,31,32,33,36,38,39\}$. Further, $i_{23}=17$, $i_{41} \in\{2,11,21\} \cup \mathcal{M}_{1} \cup \mathcal{M}_{2} \cup M \cup\{4,22,31\}$ or vice versa. Here we observe that $i_{41}$ exists since $41 \nmid d$. Thus $23 \cdot 41 \mid \prod a_{i}$ where $i$ runs through the set $\{2,11,21\} \cup \mathcal{M}_{1} \cup \mathcal{M}_{2} \cup\{4,22,31\}$. Therefore $a_{i} \in\{1,2,7,14\}$ for $i \in \mathcal{I}_{3}^{1} \cup \mathcal{I}_{3}^{2}$, where $B=\mathcal{I}_{3}^{0} \cup \mathcal{I}_{3}^{1} \cup \mathcal{I}_{3}^{2}, B=\mathcal{I}_{5}^{+} \cup \mathcal{I}_{5}^{-}$with

$$
\mathcal{I}_{3}^{0}=\{4,22,31\}, \quad \mathcal{I}_{3}^{1}=\{12,24,33,36,39\}, \quad \mathcal{I}_{3}^{2}=\{8,26,32,38\}
$$

and

$$
\mathcal{I}_{5}^{+}=\{4,24,26,31,36,39\}, \quad \mathcal{I}_{5}^{-}=\{8,12,22,32,33,38\}
$$

by taking $\mathcal{J}=B$. We get

$$
\mathcal{J}_{1}=\{24,36,39\}, \quad \mathcal{J}_{2}=\{12,33\}, \quad \mathcal{J}_{3}=\{26\}, \quad \mathcal{J}_{4}=\{8,32,38\},
$$

and $a_{24}=a_{36}=a_{39}=1, a_{12}=a_{33}=7, a_{26}=14, a_{8}=a_{32}=a_{38}=2$ by (17). Since

$$
\left(\frac{a_{i}}{41}\right)=1 \quad \text { for } a_{i} \in\{1,2\}
$$

we see that $\left(\frac{a_{i}}{41}\right)=1$ for $i \in\{8,24,32,36,38,39\}$, which is not valid by the possibilities for $i_{41}$.

All other cases are excluded similarly. Analogously to (20) and (21), we use $\left(\frac{a_{i}}{k}\right)=1$ for 


$$
a_{i} \in \begin{cases}\{1,7\} & \text { if } k=37,53,59 \\ \{1,2\} & \text { if } k=31,41,47 \\ \{1,14\} & \text { if } k=43\end{cases}
$$

to exclude the remaining possibilities.

3.6. The case $k=61$. We have $q_{1}=59, q_{2}=61$ and $\mathcal{P}_{1}=\{7,13,17,29$, $47,53\}$. Then the pairs $\left(i_{q_{1}}, i_{q_{2}}\right)$ are given by $(8,6),(9,7),(10,8),(11,9)$, i.e. $(i+2, i)$ with $6 \leq i \leq 9$.

Let $\left(i_{59}, i_{61}\right)=(8,6)$. Then $\mathcal{P}_{1}=\{7,13,17,29,47,53\}, \mathcal{M}_{1}=\{2,4,9,11$, $14,15,16,20,25,28,32,33,38,39,41,46,50,53,54,60\}, \mathcal{B}_{1}=\{0,1,3,5,7,10$, $12,13,17,18,19,21,22,23,24,26,27,29,30,31,34,35,36,37,40,42,43$, $44,45,47,48,49,51,52,55,56,57,58,59\}, i_{7}=4, i_{13}=2, i_{17}=16, i_{29}=9$ and $a_{14}, a_{20}$ are divisible by 47,53 . Further, $\operatorname{gcd}\left(p, a_{i}\right)=1$ for $i \in \mathcal{B}_{1}$ and $p \in \mathcal{P}_{1}$. Let $\mathcal{P}_{0}=\mathcal{P}_{1} \cup\{59,61\}, p_{1}=7, p_{2}=17,\left(i_{1}, i_{2}\right):=(4,16), \mathcal{I}=\mathcal{B}_{1}$, $\mathcal{P}=\mathcal{P}_{2}:=\Lambda(7,17) \backslash \mathcal{P}_{0}=\{11,19,23,37\}$ and $\ell \leq \ell_{2}=\sum_{p \in \mathcal{P}_{2}}\lceil k / p\rceil=15$. Then $2 \ell_{2}<\left|\mathcal{I}^{\prime}\right|=\left|\mathcal{B}_{1}\right|-1$. By Corollary 1 , we get $\mathcal{M}=: \mathcal{M}_{2}, \mathcal{B}=: \mathcal{B}_{2}$ such that $\left(\mathcal{M}_{2}, \mathcal{B}_{2}, \mathcal{P}_{2}, \ell_{2}\right)$ has Property $\mathfrak{H}$. We find that $\mathcal{M}_{2}=\{1,10,12$, $21,23,29,30,34,44,45,48,56\}, \mathcal{B}_{2}=\{0,3,5,7,13,17,19,22,24,26,27$, $31,35,36,37,40,42,43,47,49,51,52,55,57,58,59\}, i_{11}=1, i_{19}=10$, $i_{23}=21, i_{37}=30$. Now we take $\mathcal{P}_{0}=\mathcal{P}_{1} \cup \mathcal{P}_{2} \cup\{59,61\}, p_{1}=11$, $p_{2}=59,\left(i_{1}, i_{2}\right):=(1,8), \mathcal{I}=\mathcal{B}_{2}, \mathcal{P}=\mathcal{P}_{3}:=\Lambda(11,59) \backslash \mathcal{P}_{0}=\{31,41\}$ and $\ell \leq \ell_{3}=\sum_{p \in \mathcal{P}_{3}}\lceil k / p\rceil=4$. Then $2 \ell_{3}<\left|\mathcal{I}^{\prime}\right|=\left|\mathcal{B}_{2}\right|$. By Corollary 1, we get $\mathcal{M}=: \mathcal{M}_{3}$ and $\mathcal{B}=: \mathcal{B}_{3}$ such that $\left(\mathcal{M}_{3}, \mathcal{B}_{3}, \mathcal{P}_{3}, \ell_{3}\right)$ has Property $\mathfrak{H}$. We get $\mathcal{M}_{3}=\{0,5,26,36\}$, which cannot be covered by $\mathcal{P}_{3}$. This is a contradiction. The remaining cases are excluded similarly.

3.7. The cases $k=67,71$. We have $q_{1}=43, q_{2}=67$ and $\mathcal{P}_{1} \subseteq\{11,13$, $19,29,31,37,41,53,71\}$. Then the pairs $\left(i_{q_{1}}, i_{q_{2}}\right)$ are given by

$$
\begin{aligned}
& k=67:(i, i), 6 \leq i \leq 33 \\
& k=71:(i, i), 0 \leq i \leq 35, i \neq 24,25, \text { and }(24,0),(25,1),(26,2),(27,3) .
\end{aligned}
$$

Let $k=71$ and $\left(i_{43}, i_{67}\right)=(27,3)$. We see that $\mathcal{P}_{1}=\{11,13,19,29,31,37$, $41,53,71\}, \mathcal{M}_{1}=\{4,5,8,12,13,15,17,18,26,29,31,32,33,37,39,41,44,48$, $51,57,59\}, \mathcal{B}_{1}=\{0,1,2,6,7,9,10,11,14,16,19,20,21,22,23,24,25,28,30$, $34,35,36,38,40,42,43,45,46,47,49,50,52,53,54,55,56,58,60,61,62,63$, $64,65,66,67,68,69\}, i_{11}=4, i_{13}=5, i_{19}=13$. Therefore $\{8,12,17,29,33$, $39,41\}$ is covered by $\{29,31,37,41,53,71\}$ implying either $i_{29}=12$ or $i_{29} \in$ $\{17,29,33\}, i_{31}=8$. Let $i \in \mathcal{B}_{1}$ and $p \mid a_{i}$ with $p \in \mathcal{P}_{1}$. Then there is a $q \in \mathcal{P}_{1}$ such that $p q \mid a_{i}$ since $\mathfrak{i}\left(\mathcal{P}_{1}\right)$ is even. Next we consider the case $i_{31}=8$. Then $\{12,17,29,33,41\}=: \mathcal{M}_{1}^{\prime}$ is covered by $\{29,37,41,53,71\}$ and $i_{29} \neq 12$. For $29 \in \mathcal{M}_{1}^{\prime}$, we may suppose that either $29\left|a_{29}, 41\right| a_{17}, 29 \cdot 41 \mid a_{58}$ or $29 \mid a_{29}$, $41\left|a_{41}, 29 \cdot 41\right| a_{0}$. Thus 0 or 58 in $\mathcal{B}_{1}$ correspond to 29 . We argue as above that for any other element of $\mathcal{M}_{1}^{\prime}$, there is no corresponding element in $\mathcal{B}_{1}$. 
For the first case, we derive similarly that $31\left|a_{33}, 37\right| a_{39}, 31 \cdot 37 \mid a_{2}$ or $37\left|a_{17}, 37 \cdot 71\right| a_{54}$ or $37\left|a_{29}, 37 \cdot 71\right| a_{63}$ or $41\left|a_{17}, 37 \cdot 71\right| a_{58}$. Therefore

$$
29 \cdot 31 \cdot 37 \cdot 41 \cdot 53 \cdot 71 \mid \prod(n+i d) \quad \text { for } i \in \mathcal{M}_{1} \cup\{3,27,70\} \cup \mathcal{B}_{1}^{\prime}
$$

where $\mathcal{B}_{1}^{\prime}=\{2,54,58,63\}$ if $i_{29}=12$ and $\{0,58\}$ otherwise. Further,

$$
i_{71} \in \mathcal{M}_{1} \cup\{27\} \cup \mathcal{B}_{1}^{\prime} \text { and } i_{71} \neq 32 .
$$

For each possibility $i_{29} \in\{0,4,12,17\}$, we now take $\mathcal{P}_{0}=\mathcal{P}_{1} \cup\{43,67\}$, $p_{1}=19, p_{2}=29,\left(i_{1}, i_{2}\right):=\left(13, i_{29}\right), \mathcal{I}=\mathcal{B}_{1} \backslash \mathcal{B}_{1}^{\prime}, \mathcal{P}=\mathcal{P}_{2}:=\Lambda(19,29) \backslash \mathcal{P}_{0}=$ $\{17,47,59,61\}$ and $\ell=\ell_{2}=\sum_{p \in \mathcal{P}_{2}}\lceil k / p\rceil=11$. Then $\left|\mathcal{I}^{\prime}\right|=\left|\mathcal{B}_{1}\right|-4>2 \ell_{2}$. Thus the conditions of Corollary 1 are satisfied and we get $\mathcal{M}=: \mathcal{M}_{2}$ and $\mathcal{B}=: \mathcal{B}_{2}$ with $\left(\mathcal{M}_{2}, \mathcal{B}_{2}, \mathcal{P}_{2}, \ell_{2}\right)$ having Property $\mathfrak{H}$. We check that $\left|\mathcal{M}_{2}\right| \leq \ell_{2}$ only at $i_{29}=12$, in which case we get $\mathcal{M}_{2}=\{9,11,19,23,36,53\}, \mathcal{B}_{2}=\{0,1$, $6,7,10,14,16,20,21,22,24,25,28,30,34,35,38,40,42,43,45,46,47,49$, $50,52,55,56,60,61,62,63,64,65,67,68,69\}, i_{17}=2$ and $\{9,11,23\}$ is covered by $\{47,59,61\}$. Thus $47.59 \cdot 61 \mid a_{9} a_{11} a_{23}$. Further, $p \nmid a_{i}$ for $i \in \mathcal{B}_{2}$ and $p \in \mathcal{P}_{2}$. We now take $\mathcal{P}_{0}=\mathcal{P}_{1} \cup \mathcal{P}_{2} \cup\{43,67\}, p_{1}=11, p_{2}=13,\left(i_{1}, i_{2}\right):=(4,5), \mathcal{I}=$ $\mathcal{B}_{2}, \mathcal{P}=\mathcal{P}_{3}:=\Lambda(11,13) \backslash \mathcal{P}_{0}=\{5\}$ and $\ell=\ell_{3}=\lceil k / 5\rceil=15$. Then $\left|\mathcal{I}^{\prime}\right|=\left|\mathcal{B}_{2}\right|$ $>2 \ell_{3}$. By Corollary 1 , we get $\mathcal{M}=: \mathcal{M}_{3}$ and $\mathcal{B}=: \mathcal{B}_{3}$ such that $\left(\mathcal{M}_{3}\right.$, $\left.\mathcal{B}_{3}, \mathcal{P}_{3}, \ell_{3}\right)$ has Property $\mathfrak{H}$. We calculate $\mathcal{M}_{3}=\{0,10,25,30,35,40,50,55$, $60,65\}, \mathcal{B}_{3}=\{1,6,7,14,16,20,21,22,24,28,34,38,42,43,45,46,47,49$, $52,54,56,58,61,62,63,64,66,67,68,69\}, i_{5}=0$ and further $5 \nmid a_{20} a_{45}$. Lastly, we take $\mathcal{P}_{0}=\mathcal{P}_{1} \cup \mathcal{P}_{2} \cup \mathcal{P}_{3} \cup\{43,67\}, p_{1}=5, p_{2}=11,\left(i_{1}, i_{2}\right):=(0,4)$, $\mathcal{I}=\mathcal{B}_{3}, \mathcal{P}=\mathcal{P}_{4}:=\Lambda(5,11) \backslash \mathcal{P}_{0}=\{3,23\}$ and $\ell=\ell_{4}=\sum_{p \in \mathcal{P}_{4}}\lceil k / p\rceil$. By Lemma 5 , we see that $M=\{16,22,24,28,43,46,47,49,64,67\}$ is covered by $\mathcal{P}_{4}, i_{3}=i_{23}=1, B=\{1,6,7,14,21,34,38,42,52,56,61,62,63,68,69\}$ and hence $3 \nmid a_{7} a_{34} a_{52} a_{61}$ and possibly $3 \cdot 23 \mid a_{1}$. Therefore $a_{i} \in\{1,2,7,14\}$ for $i \in B \backslash\{1\}$. By taking $\mathcal{J}=B \backslash\{1\}$, we have $B \backslash\{1\}=\mathcal{I}_{3}^{0} \cup \mathcal{I}_{3}^{1} \cup \mathcal{I}_{3}^{-}=\mathcal{I}_{5}^{+} \cup \mathcal{I}_{5}^{-}$ with

$$
\mathcal{I}_{3}^{0}=\{7,34,52,61\}, \quad \mathcal{I}_{3}^{1}=\{6,21,42,63,69\}, \quad \mathcal{I}_{3}^{-}=\{14,38,56,62,68\}
$$

and

$$
\mathcal{I}_{5}^{+}=\{6,14,21,34,56,61,69\}, \quad \mathcal{I}_{5}^{-}=\{7,38,42,52,62,63,68\} .
$$

Therefore

$$
\mathcal{J}_{1}=\{6,21,69\}, \quad \mathcal{J}_{2}=\{42,63\}, \quad \mathcal{J}_{3}=\{14,56\}, \quad \mathcal{J}_{4}=\{38,62,68\},
$$

and hence $a_{6}=a_{21}=a_{69}=1, a_{42}=a_{63}=7, a_{14}=a_{56}=14, a_{38}=$ $a_{62}=a_{68}=2$ by (17). Further, we get $a_{34}=a_{61}=1$ and $a_{52}=2$ by taking residue classes modulo 5 . Since $\left(\frac{1}{71}\right)=\left(\frac{2}{71}\right)=1$, we see that $\left(\frac{a_{i}}{71}\right)=1$ for $i \in\{6,21,34,38,52,61,62,68,69\}$, which is not valid by the possibilities for $i_{71}$ given by $(22)$. 
Let $k=67$ and $\left(i_{43}, i_{67}\right)=(9,9)$. We see that $\mathcal{P}_{1}=\{11,13,19,29,31,37$, $41,53\}, \mathcal{M}_{1}=\{20,22,28,31,35,38,40,42,46,47,48,50,53,61,62,64,66\}$, $\mathcal{B}_{1}=\{0,1,2,3,4,5,6,7,8,10,11,12,13,14,15,16,17,18,19,21,23,24,25,26$, $27,29,30,32,33,34,36,37,39,41,43,44,45,49,51,54,55,56,57,58,59,60$, $63,65\}, i_{11}=i_{13}=i_{19}=9$ and $\{38,40,46,50,62\}$ is covered by $\{29,31,37$, $41,53\}$. Further, $p \nmid a_{i}$ for $i \in \mathcal{B}_{1}$ and $p \in \mathcal{P}_{1}$ except possibly when $29 \mid a_{50}$, $41\left|a_{62}, 29 \cdot 41\right| a_{21}$. Now we take $\mathcal{P}_{0}=\mathcal{P}_{1} \cup\{43,67\}, p_{1}=11, p_{2}=13$, $\left(i_{1}, i_{2}\right):=(9,9), \mathcal{I}=\mathcal{B}_{1} \backslash\{21\}$ and $\mathcal{P}=\mathcal{P}_{2}:=\Lambda(11,13) \backslash \mathcal{P}_{0}=\{5,17,47$, $59,61\}$. If $5 \nmid d$, we observe that there is at least one multiple of 5 among $n+\left(i_{11}+11 i\right) d, 0 \leq i \leq 5$, and $\ell \leq \sum_{p \in \mathcal{P}_{2}}\lceil k / p\rceil-1=23$. Thus we always have $\ell \leq 23=\ell_{2}$. Then $\left|\mathcal{I}^{\prime}\right|=\left|\mathcal{B}_{1}\right|-1>2 \ell_{2}$ since $\left|B_{1}\right|=48$. Thus the conditions of Corollary 1 are satisfied and we get $\mathcal{M}=: \mathcal{M}_{2}, \mathcal{B}=: \mathcal{B}_{2}$ such that $\left(\mathcal{M}_{2}, \mathcal{B}_{2}, \mathcal{P}_{2}, \ell_{2}\right)$ has Property $\mathfrak{H}$. We have $\mathcal{M}_{2}=\{0,1,2,3,5,6,7,8,14,19$, $24,26,29,39,43,44,49,54,56,60\}$, which cannot be covered by $\mathcal{P}_{2}$. This is a contradiction. The cases $k=67,\left(i_{43}, i_{67}\right)=(i, i)$ with $9 \leq i \leq 28$, and $k=71,\left(i_{43}, i_{67}\right)=(i, i)$ with $13 \leq i \leq 28, i \neq 24,25$, are excluded in the same way as in this paragraph. The remaining cases are excluded in the same way as $k=71,\left(i_{43}, i_{67}\right)=(27,3)$ given in the preceding paragraph.

3.8. The cases $k=73,79$. We have $q_{1}=23, q_{2}=73$ and $\mathcal{P}_{1} \subseteq\{13,19,29$, $31,37,47,59,61,67,79\}$. Then the pairs $\left(i_{q_{1}}, i_{q_{2}}\right)$ are given by

$$
\begin{aligned}
k=73: & (6,2),(7,3),(8,4),(9,5) ; \\
k=79: & (0,0),(1,1),(2,2),(7,3),(8,4),(9,5),(10,6),(11,7),(12,8), \\
& (13,9),(14,10),(15,11),(16,12),(17,13),(18,14),(19,15) .
\end{aligned}
$$

These pairs are of the form $(i+4, i)$ except for $(0,0),(1,1),(2,2)$ in the case $k=79$.

Let $k=79$ and $\left(i_{23}, i_{73}\right)=(8,4)$. We see that $\mathcal{P}_{1}=\{13,19,29,31,37,47$, $59,61,67,79\}, \mathcal{M}_{1}=\{1,3,10,12,15,16,18,19,20,25,30,38,39,40,46,48$, $51,58,64,78\}, \mathcal{B}_{1}=\{0,2,5,6,7,9,11,13,14,17,21,22,23,24,26,27$, $28,29,32,33,34,35,36,37,41,42,43,44,45,47,49,50,52,53,55,56$, $57,59,60,61,62,63,65,66,67,68,69,70,71,72,73,74,75,76\}, i_{13}=12$, $i_{19}=1$ and $\{3,10,15,16,18,19,30,40,46,48,78\}$ is covered by $\{29,31,37$, $47,59,61,67,79\}$. Thus

$$
29 \cdot 31 \cdot 37 \cdot 47 \cdot 59 \cdot 61 \cdot 67 \cdot 79 \mid \prod(n+i d)
$$

for $i \in\{3,10,15,16,18,19,30,40,46,48,78\}$.

Further, we have

$$
i_{79} \in\{10,15,16,18,19,30,40,46,48\}
$$

and either $i_{29}=19$ or $i_{29} \in\{1,10,16,18\}, i_{31}=15, i_{37}=3, i_{59}=19$. Also, for $p \in \mathcal{P}_{1}$, we have $p \nmid a_{i}$ for $i \in \mathcal{B}_{1}$ since $\mathfrak{i}\left(\mathcal{P}_{1}\right)$ is even for $i \in \mathcal{B}_{1}$. 
For each possibility $i_{29} \in\{1,10,16,18,19\}$, we now take $\mathcal{P}_{0}=\mathcal{P}_{1} \cup\{23,73\}$, $p_{1}=19, p_{2}=29,\left(i_{1}, i_{2}\right):=\left(1, i_{29}\right), \mathcal{I}=\mathcal{B}_{1}, \mathcal{P}=\mathcal{P}_{2}:=\Lambda(19,29) \backslash \mathcal{P}_{0}$ $=\{11,17,43,53,71\}$ and $\ell=\ell_{2}=\sum_{p \in \mathcal{P}_{2}}\lceil k / p\rceil=19$. Then $\left|\mathcal{I}^{\prime}\right| \geq\left|\mathcal{B}_{1}\right|-2$ $>2 \ell_{2}$. Thus the conditions of Corollary 1 are satisfied and we have $\mathcal{M}=$ : $\mathcal{M}_{2}, \mathcal{B}=: \mathcal{B}_{2}$ such that $\left(\mathcal{M}_{2}, \mathcal{B}_{2}, \mathcal{P}_{2}, \ell_{2}\right)$ has Property $\mathfrak{H}$, implying $i_{29}=19$, in which case we get $\mathcal{M}_{2}=\{0,6,9,11,22,24,26,33,34,43,44,55,60,66\}$, $\mathcal{B}_{2}=\{2,5,7,13,14,17,21,23,27,28,29,32,35,36,37,41,42,45,47,49,50$, $52,53,56,57,59,61,62,63,65,67,68,69,70,71,72,73,74,75,76\}, i_{11}=0$, $i_{17}=9$ and $\{6,24,34\}$ is covered by $\{43,53,71\}$. Thus $43 \cdot 53 \cdot 71 \mid a_{6} a_{24} a_{34}$. Further, $p \nmid a_{i}$ for $i \in \mathcal{B}_{2}$ and $p \in \mathcal{P}_{2}$. We now take $\mathcal{P}_{0}=\mathcal{P}_{1} \cup \mathcal{P}_{2} \cup\{23,73\}$, $p_{1}=11, p_{2}=13,\left(i_{1}, i_{2}\right):=(0,12), \mathcal{I}=\mathcal{B}_{2}, \mathcal{P}=\mathcal{P}_{3}:=\Lambda(11,13) \backslash \mathcal{P}_{0}=\{5\}$ and $\ell=\ell_{3}=\lceil k / 5\rceil=16$. Then $\left|\mathcal{I}^{\prime}\right|=\left|\mathcal{B}_{2}\right|>2 \ell_{3}$. By Corollary 1 , we get $\mathcal{M}=: \mathcal{M}_{3}$ and $\mathcal{B}=: \mathcal{B}_{3}$ with $\left(\mathcal{M}_{3}, \mathcal{B}_{3}, \mathcal{P}_{3}, \ell_{3}\right)$ having Property $\mathfrak{H}$. We calculate $\mathcal{M}_{3}=\{7,17,32,37,42,47,57,62,67,72\}, \mathcal{B}_{3}=\{2,5,13,14,21,23,27$, $28,29,35,36,41,45,49,50,52,53,56,59,61,63,65,68,69,70,71,73,74$, $75,76\}, i_{5}=2$ and $5 \nmid a_{i}$ for $i \in \mathcal{B}_{3}$. Lastly, we take $\mathcal{P}_{0}=\mathcal{P}_{1} \cup \mathcal{P}_{2} \cup$ $\mathcal{P}_{3} \cup\{23,73\}, p_{1}=5, p_{2}=11,\left(i_{1}, i_{2}\right):=(2,0), \mathcal{I}=\mathcal{B}_{3}, \mathcal{P}=\mathcal{P}_{4}:=$ $\Lambda(5,11) \backslash \mathcal{P}_{0}=\{3,41\}$ and $\ell=\ell_{4}=\sum_{p \in \mathcal{P}_{4}}\lceil k / p\rceil$. By Lemma 5 , we see that $M=\{23,29,35,36,50,53,56,65,71,74\}$ is covered by $\mathcal{P}_{4}, i_{3}=2$, $i_{41}=36, B=\{5,13,14,21,28,41,45,49,59,61,63,68,69,70,73,75,76\}$ and hence $a_{i} \in\{1,2,7,14\}$ for $i \in B$. By taking $\mathcal{J}=B$, we have $B=\mathcal{I}_{3}^{0} \cup \mathcal{I}_{3}^{1}$ $\cup \mathcal{I}_{3}^{2}=\mathcal{I}_{5}^{+} \cup \mathcal{I}_{5}^{-}$with

$$
\begin{gathered}
\mathcal{I}_{3}^{0}=\{5,14,41,59,68\}, \quad \mathcal{I}_{3}^{1}=\{13,28,49,61,70,73,76\}, \\
\mathcal{I}_{3}^{2}=\{21,45,63,69,75\}
\end{gathered}
$$

and

$\mathcal{I}_{5}^{+}=\{13,21,28,41,61,63,68,73,76\}, \quad \mathcal{I}_{5}^{-}=\{5,14,45,49,59,69,70,75\}$.

Thus

$\mathcal{J}_{1}=\{13,28,61,73,76\}, \quad \mathcal{J}_{2}=\{49,70\}, \quad \mathcal{J}_{3}=\{21,63\}, \quad \mathcal{J}_{4}=\{45,69,75\}$, and hence $a_{13}=a_{28}=a_{61}=a_{73}=a_{76}=1, a_{49}=a_{70}=7, a_{21}=a_{63}=14$, $a_{45}=a_{69}=a_{75}=2$ by (17). Further, we get $a_{41}=a_{68}=1$ and $a_{5}=a_{59}=2$ by residues modulo 5 . Since $\left(\frac{1}{79}\right)=\left(\frac{2}{79}\right)=1$, we see that $\left(\frac{a_{i}}{71}\right)=1$ for $i \in$ $\{5,13,28,41,45,59,61,68,69,75,76\}$, which is not valid by the possibilities for $i_{79}$ given by (23). The other cases are excluded similarly.

3.9. The case $k=83$. We have $q_{1}=37, q_{2}=83$ and $\mathcal{P}_{1}=\{17,23,29,31$, $47,53,59,61,67,71,73\}$. Then the pairs $\left(i_{q_{1}}, i_{q_{2}}\right)$ are given by

$$
\begin{aligned}
& (13,4),(14,5),(15,6),(16,7),(17,8),(18,9),(19,10), \\
& (20,11),(21,12),(22,13),(23,14),(24,15),(25,16),(26,17) .
\end{aligned}
$$

These pairs are of the form $(i+9, i)$ with $4 \leq i \leq 17$. 
Let $\left(i_{37}, i_{83}\right)=(13,4)$. We see that $\mathcal{P}_{1}=\{17,23,29,31,47,53,59,61,67$, $71,73\}, \mathcal{M}_{1}=\{0,2,14,16,18,19,20,25,26,28,29,34,36,40,41,53,56$, $58,64,70\}, \mathcal{B}_{1}=\{1,3,5,6,7,8,9,10,11,12,15,17,21,22,23,24,27,30,31$, $32,33,35,37,38,39,42,43,44,45,46,47,48,49,51,52,54,55,57,59,60$, $61,62,63,65,66,67,68,69,71,72,73,74,75,76,77,78,79,80,81,82\}$, $i_{17}=2, i_{23}=18, i_{29}=0, i_{31}=25$ and $\{14,16,20,26,28,34,40\}$ is covered by $\{47,53,59,61,67,71,73\}$. Further, $p \nmid a_{i}$ for $i \in \mathcal{B}_{1}$ and $p \in \mathcal{P}_{1}$. For each possibility $i_{73} \in\{14,16,20,26,28,34,40\}$, we take $\mathcal{P}_{0}=\mathcal{P}_{1} \cup\{37,83\}, p_{1}=$ $23, p_{2}=73,\left(i_{1}, i_{2}\right):=\left(18, i_{73}\right), \mathcal{I}=\mathcal{B}_{1}, \mathcal{P}=\mathcal{P}_{2}:=\Lambda(23,73) \backslash \mathcal{P}_{0}=$ $\{13,19,79\}$ and $\ell=\ell_{2}=\sum_{p \in \mathcal{P}_{2}}\lceil k / p\rceil=14$. Then $\left|\mathcal{I}^{\prime}\right|=\left|\mathcal{B}_{1}\right|>2 \ell_{2}$. Thus the conditions of Corollary 1 are satisfied and we get $\mathcal{M}=: \mathcal{M}_{2}, \mathcal{B}=: \mathcal{B}_{2}$ such that $\left(\mathcal{M}_{2}, \mathcal{B}_{2}, \mathcal{P}_{2}, \ell_{2}\right)$ has Property $\mathfrak{H}$, which is possible only if $i_{73}=14$. Then $\mathcal{M}_{2}=\{8,9,11,22,30,35,48,49,61,68,74\}$. Therefore $i_{13}=9, i_{19}=11$ and $i_{79}=8$. This is not possible by applying the case $k=73$ to $(n+9 d) \cdots(n+$ $81 d)$. Similarly, for $\left(i_{37}, i_{83}\right)=(14,5)$, we get $i_{73}=15, i_{79}=9$ and this is excluded by applying the case $k=73$ to $(n+10 d) \cdots(n+82 d)$. For all the remaining cases, we continue similarly to find that $\mathcal{M}_{2}$ is not covered by $\mathcal{P}_{2}$ for the possible choices of $i_{73}$, and hence they are excluded.

3.10. The case $k=89$. We have $q_{1}=79, q_{2}=89$ and $\mathcal{P}_{1}=\{13,17,19$, $23,31,47,53,71,83\}$. Then the pairs $\left(i_{q_{1}}, i_{q_{2}}\right)$ are given by

$$
(16,6),(17,7),(18,8),(19,9),(20,10),(21,11) .
$$

These pairs are of the form $(i+10, i)$ with $6 \leq i \leq 11$.

Let $\left(i_{79}, i_{89}\right)=(16,6)$. We see that $\mathcal{P}_{1}=\{13,17,19,23,31,47,53,71,83\}$, $\mathcal{M}_{1}=\{0,1,2,3,4,10,12,17,19,24,26,27,30,33,38,42,43,44,48,49,56$, $57,61,64,69,72,76,78,82\}, \mathcal{B}_{1}=\{5,7,8,9,11,13,14,15,18,20,21,22,23$, $25,28,29,31,32,34,35,36,37,39,40,41,45,46,47,50,51,52,53,54,55$, $58,59,60,62,63,65,66,67,68,70,71,73,74,75,77,79,80,81,83,84,85$, $86,87,88\}, i_{13}=4, i_{17}=10, i_{19}=0, i_{23}=3, i_{31}=2, i_{47}=1$ and $\{12,24,42\}$ is covered by $\{53,71,83\}$. Further, $p \nmid a_{i}$ for $i \in \mathcal{B}_{1}$ and $p \in \mathcal{P}_{1}$. Now we take $\mathcal{P}_{0}=\mathcal{P}_{1} \cup\{79,89\}, p_{1}=31, p_{2}=89,\left(i_{1}, i_{2}\right):=(2,6), \mathcal{I}=\mathcal{B}_{1}$ and $\mathcal{P}=\mathcal{P}_{2}:=\Lambda(31,89) \backslash \mathcal{P}_{0}=\{7,11,41,59,73\}$. If $7 \nmid d$, we observe that there is at least one multiple of 7 among $n+\left(i_{13}+13 i\right) d, 0 \leq i \leq 6$, and $\ell \leq \ell_{2}=\sum_{p \in \mathcal{P}_{2}}\lceil k / p\rceil-1=28$. Thus in all cases, we have $\ell \leq \ell_{2}$ and $\left|\mathcal{I}^{\prime}\right|=\left|\mathcal{B}_{1}\right|>2 \ell_{2}$. Therefore the conditions of Corollary 1 are satisfied and we get $\mathcal{M}=: \mathcal{M}_{2}$ and $\mathcal{B}=: \mathcal{B}_{2}$ with $\left(\mathcal{M}_{2}, \mathcal{B}_{2}, \mathcal{P}_{2}, \ell_{2}\right)$ having Property $\mathfrak{H}$. We find $\mathcal{M}_{2}=\{7,11,13,22,25,29,32,36,39,40,51,53,54,60,62,67,73,74,81$, $84,88\}, \mathcal{B}_{2}=\{5,8,9,14,15,18,20,21,23,28,31,34,35,37,41,45,46,47$, $50,52,55,58,59,63,65,66,68,70,71,75,77,79,80,83,85,86,87\}, i_{7}=4$, $i_{11}=7, i_{41}=13$ and $\{22,36\}$ is covered by $\{59,73\}$. Further, for $p \in \mathcal{P}_{2}, p \nmid a_{i}$ for $i \in \mathcal{B}_{2} \backslash\{18\}$. We take $\mathcal{P}_{0}=\mathcal{P}_{1} \cup \mathcal{P}_{2} \cup\{79,89\}, p_{1}=41, p_{2}=79,\left(i_{1}, i_{2}\right):=$ $(13,16), \mathcal{I}=\mathcal{B}_{2} \backslash\{18\}, \mathcal{P}=\mathcal{P}_{3}:=\Lambda(41,79) \backslash \mathcal{P}_{0}=\{37,43,61,67\}$ and $\ell=$ 
$\ell_{3}=\sum_{p \in \mathcal{P}_{3}}\lceil k / p\rceil=10$. Then $\left|\mathcal{I}^{\prime}\right|=|\mathcal{I}|=\left|\mathcal{B}_{2}\right|-1>2 \ell_{3}$. Thus the conditions of Corollary 1 are satisfied and we have $\mathcal{M}=: \mathcal{M}_{3}, \mathcal{B}=: \mathcal{B}_{3}$ such that $\left(\mathcal{M}_{3}, \mathcal{B}_{3}, \mathcal{P}_{3}, \ell_{3}\right)$ has Property $\mathfrak{H}$. We get $\mathcal{M}_{3}=\{9,21,28,34,52,58\}, \mathcal{B}_{3}=$ $\{5,8,14,15,20,23,31,35,37,41,45,46,47,50,55,59,63,65,66,68,70,71$, $75,77,79,80,83,85,86,87\}, i_{37}=21, i_{43}=9$ and $\{28,34\}$ is covered by $\{61,67\}$. Therefore $p \in\{2,3,5,29\}$ whenever $p \mid a_{i}$ for $i \in \mathcal{B}_{3}$. Now we take $\mathcal{P}_{0}=\mathcal{P}_{1} \cup \mathcal{P}_{2} \cup \mathcal{P}_{3} \cup\{79,89\}, p_{1}=7, p_{2}=17,\left(i_{1}, i_{2}\right):=(4,10), \mathcal{I}=\mathcal{B}_{3}$, $\mathcal{P}=\mathcal{P}_{4}:=\Lambda(7,17) \backslash \mathcal{P}_{0}=\{29\}$ and $\ell=\ell_{4}=\lceil k / 29\rceil=4$. Then $\left|\mathcal{I}^{\prime}\right|=\left|\mathcal{B}_{3}\right|-1$ since $46 \in \mathcal{B}_{3}$ and $\left|\mathcal{B}_{3}\right|-1>2 \ell_{3}$. By Corollary 1 , we get $\mathcal{M}=: \mathcal{M}_{4}$ and $\mathcal{B}=$ : $\mathcal{B}_{4}$ with $\left(\mathcal{M}_{4}, \mathcal{B}_{4}, \mathcal{P}_{4}, \ell_{4}\right)$ having Property $\mathfrak{H}$. We find $\mathcal{M}_{4}=\{8,37,66\}, \mathcal{B}_{4}=$ $\{5,14,15,20,23,31,35,41,45,47,50,55,59,63,65,68,70,71,75,77,79,80$, $83,85,86,87\}, i_{29}=8$ and $P\left(a_{i}\right) \leq 5$ for $i \in \mathcal{B}_{4}$. Now we get a contradiction by taking $k=6$ and $(n+47 d)(n+55 d)(n+63 d)(n+71 d)(n+79 d)(n+87 d)=$ $b^{\prime} y^{\prime 2}$. Similarly the pair $\left(i_{79}, i_{89}\right)=(17,7)$ is excluded by applying $k=6$ to $(n+48 d)(n+56 d)(n+64 d)(n+72 d)(n+80 d)(n+88 d)$. For all the remaining cases, we continue similarly to find that $\mathcal{M}_{3}$ is not covered by $\mathcal{P}_{3}$, and hence they are excluded.

4. Proof of Lemma 7. Assume that $Q_{1} \nmid d$ and $Q_{2} \nmid d$. Then, by taking the mirror image (4) of (2), there is no loss of generality in assuming that $0 \leq i_{Q_{1}}<Q_{1}, 0 \leq i_{Q_{2}} \leq \min \left(Q_{2}-1,(k-1) / 2\right)$. Further, $i_{Q_{2}} \geq k-k^{\prime}$ if $Q_{2}=k$. Let $\mathcal{P}_{0}=\left\{Q_{0}\right\}, p_{1}=Q_{1}, p_{2}=Q_{2},\left(i_{1}, i_{2}\right):=\left(i_{Q_{1}}, i_{Q_{2}}\right), \mathcal{I}=[0, k) \cap \mathbb{Z}$ and $\mathcal{P}=\mathcal{P}_{1}:=\Lambda\left(Q_{1}, Q_{2}\right) \backslash \mathcal{P}_{0}$. Then $\left|\mathcal{I}^{\prime}\right| \geq k-\left\lceil k / Q_{1}\right\rceil-\left\lceil k / Q_{2}\right\rceil$ and $\ell \leq \ell_{1}$ where $\ell_{1}=\sum_{p \in \mathcal{P}_{1}}\lceil k / p\rceil$. In fact we can take $\ell_{1}=\sum_{p \in \mathcal{P}_{1}}\lceil k / p\rceil-1$ if $\left(k, Q_{0}\right)=(79,23)$ or $\left(k, Q_{0}\right)=(59,29)$ with $i_{7} \leq 2$ by considering multiples of 13,11 or $19,7,11$, respectively.

Let $\left(k, Q_{0}\right) \neq(79,73)$. Then $\ell_{1}<\frac{1}{2}\left|\mathcal{I}^{\prime}\right|$. We observe that $\mathfrak{i}\left(\mathcal{P}_{0}\right)=0$ for $i \in \mathcal{I}^{\prime}$ since $Q_{0} \mid d$, and by Corollary 1 , we get $\mathcal{M}=: \mathcal{M}_{1}, \mathcal{B}=: \mathcal{B}_{1}$ such that $\left(\mathcal{M}_{1}, \mathcal{B}_{1}, \mathcal{P}_{1}, \ell_{1}\right)$ has Property $\mathfrak{H}$. We now restrict to all such pairs $\left(i_{Q_{1}}, i_{Q_{2}}\right)$ with $\left|\mathcal{M}_{1}\right| \leq \ell_{1}$ and $\mathcal{M}_{1}$ covered by $\mathcal{P}_{1}$. These pairs are given by

\begin{tabular}{cccc}
\hline$k$ & $Q_{0}$ & $\left(Q_{1}, Q_{2}\right)$ & $\left(i_{Q_{1}}, i_{Q_{2}}\right)$ \\
\hline 29 & 19 & $(7,17)$ & $(0,0),(0,11)$ \\
37 & 19 or 29 & $(7,17)$ & $(0,0),(1,2)$ \\
47 & 29 & $(7,17)$ & $(0,0),(4,12)$ \\
59 & 29 & $(7,17)$ & $(1,1),(1,6)$ \\
71 & 43 & $(53,67)$ & $(0,0)$ \\
89 & 79 & $(23,73)$ & $(0,0),(19,15)$ \\
\hline
\end{tabular}

Let $\left(k, Q_{0}\right)=(79,73)$ and $\left(Q_{1}, Q_{2}\right)=(53,67)$. We apply Lemma 5 to derive that either $\left|\mathcal{I}_{1}\right| \leq \ell_{1}, \mathcal{I}_{1}$ is covered by $\mathcal{P}_{1}, \mathfrak{i}\left(\mathcal{P}_{1}\right)$ is even for $i \in \mathcal{I}_{2}$, or 
$\left|\mathcal{I}_{2}\right| \leq \ell_{1}, \mathcal{I}_{2}$ is covered by $\mathcal{P}_{1}, \mathfrak{i}\left(\mathcal{P}_{1}\right)$ is even for $i \in \mathcal{I}_{1}$. We compute $\mathcal{I}_{1}, \mathcal{I}_{2}$ and we find that both $\mathcal{I}_{1}$ and $\mathcal{I}_{2}$ are not covered by $\mathcal{P}_{1}$ for each pair $\left(i_{53}, i_{67}\right)$ with $0 \leq i_{53}<53,0 \leq i_{67} \leq(k-1) / 2$.

Let $\left(k, Q_{0}\right)=(37,29),\left(Q_{1}, Q_{2}\right)=(7,17)$ and $\left(i_{7}, i_{17}\right)=(1,2)$. Then $\mathcal{P}_{1}=\{11,13,19,23,37\}$. We find that $\mathcal{M}_{1}=\{3,7,10,13,14,17,23,25\}$, $\mathcal{B}_{1}=\{0,4,5,6,9,11,12,16,18,20,21,24,26,27,28,30,31,32,33,34,35\}$, $i_{11}=3, i_{13}=10$ and $\{7,13,17\}$ is covered by $\{19,23,37\}$. Further, $p \nmid a_{i}$ for $p \in \mathcal{P}_{1}, i \in \mathcal{B}_{1}$. Now we take $\mathcal{P}_{0}=\mathcal{P}_{1} \cup\{7,17,29\}, p_{1}=11, p_{2}=13$, $\left(i_{1}, i_{2}\right):=(3,10), \mathcal{I}=\mathcal{B}_{1}, \mathcal{P}=\mathcal{P}_{2}:=\Lambda(11,13) \backslash \mathcal{P}_{0}=\{5,31\}$ and $\ell=\ell_{2}=\sum_{p \in \mathcal{P}_{2}}\lceil k / p\rceil=10$. Thus $\left|\mathcal{I}^{\prime}\right|=|\mathcal{I}|=\left|\mathcal{B}_{1}\right|=21>2 \ell_{2}$. Then the conditions of Corollary 1 are satisfied and we have $\mathcal{M}=: \mathcal{M}_{2}, \mathcal{B}=: \mathcal{B}_{2}$ such that $\left(\mathcal{M}_{2}, \mathcal{B}_{2}, \mathcal{P}_{2}, \ell_{2}\right)$ has Property $\mathfrak{H}$. We get $\mathcal{M}_{2}=\{5,6,16,21,26,31\}$, $\mathcal{B}_{2}=\{0,4,9,11,12,18,20,24,27,28,30,32,33,34,35\}, i_{5}=1,31 \mid a_{5}$ and $5 \nmid a_{11}$. Also, $P\left(a_{i}\right) \leq 3$ for $i \in B_{2}$ and $P\left(a_{31}\right)=5$. Thus $P\left(a_{30} a_{31} \cdots a_{35}\right) \leq 5$ and this is excluded by the case $k=6$. The other cases for $k=29,37,47$ are excluded similarly. Each possibility is excluded by the case $k=6$ after showing $P\left(a_{1} a_{2} \cdots a_{6}\right) \leq 5$ when $\left(k, Q_{0}\right) \in\{(29,19),(37,19),(37,29),(47,29)\}$, $\left(i_{7}, i_{17}\right)=(0,0) ; P\left(a_{22} a_{23} \cdots a_{27}\right) \leq 5$ when $\left(k, Q_{0}\right)=(29,19),\left(i_{7}, i_{17}\right)=$ $(0,11) ; P\left(a_{30} a_{31} \cdots a_{35}\right) \leq 5$ when $\left(k, Q_{0}\right)=(37,19),\left(i_{7}, i_{17}\right)=(1,2) ;$ and $P\left(a_{40} a_{41} \cdots a_{45}\right) \leq 5$ when $\left(k, Q_{0}\right)=(47,29),\left(i_{7}, i_{17}\right)=(4,12)$.

Let $\left(k, Q_{0}\right)=(59,29),\left(Q_{1}, Q_{2}\right)=(7,17)$ and $\left(i_{7}, i_{17}\right)=(1,1)$. Then $\mathcal{P}_{1}=\{11,13,19,23,37,47,59\}$. We find that $\mathcal{M}_{1}=\{0,12,14,20,23,24,27$, $30,34,38,39,40,45,47,48,53,56,58\}, \mathcal{B}_{1}=\{2,3,4,5,6,7,9,10,11,13,16$, $17,19,21,25,26,28,31,32,33,37,41,42,44,46,49,51,54,55\}, i_{11}=i_{13}=i_{19}$ $=i_{23}=1$ and $\{30,38,48\}$ is covered by $\{37,47,59\}$. Further, $p \nmid a_{i}$ for $p \in \mathcal{P}_{1}$, $i \in \mathcal{B}_{1}$. Now we take $\mathcal{P}_{0}=\mathcal{P}_{1} \cup\{7,17,29\}, p_{1}=11, p_{2}=13,\left(i_{1}, i_{2}\right):=(1,1)$, $\mathcal{I}=\mathcal{B}_{1}, \mathcal{P}=\mathcal{P}_{2}:=\Lambda(11,13) \backslash \mathcal{P}_{0}=\{5,31,43\}$ and $\ell=\ell_{2}=\sum_{p \in \mathcal{P}_{2}}\lceil k / p\rceil$. By Lemma 5 , we get $M=\{6,11,16,21,31,32,41,44,46\}, i_{5}=1,31 \cdot 43 \mid a_{32} a_{44}$, and $\mathfrak{i}\left(\mathcal{P}_{2}\right)$ is even for $i \in B=\{2,3,4,5,7,9,10,13,17,19,25,26,28,33,37$, $42,49,51,54,55\}$. Further, for $p \in \mathcal{P}_{2}, p \nmid a_{i}$ for $i \in B$. Finally we apply Lemma 5 with $\mathcal{P}_{0}=\mathcal{P}_{1} \cup \mathcal{P}_{2} \cup\{7,17,29\}, p_{1}=5, p_{2}=11,\left(i_{1}, i_{2}\right):=$ $(1,1), \mathcal{I}=B$ and $\mathcal{P}=\mathcal{P}_{3}:=\Lambda(5,11) \backslash \mathcal{P}_{0}=\{3,41,53\}$. We get $M_{1}=$ $\{4,7,13,25,28,42,49,54,55\}$, which is covered by $\mathcal{P}_{3}, i_{3}=1,\{42,54\}$ is covered by $\{41,53\}$ and $\mathfrak{i}\left(\mathcal{P}_{3}\right)$ is even for $i \in B_{1}=\{2,3,5,9,10,17,19,33,37\}$. Hence $P\left(a_{i}\right) \leq 2$ for $i \in B_{1}$. Since $\left(\frac{a_{i}}{29}\right)=\left(\frac{n}{29}\right)$ and $\left(\frac{2}{29}\right) \neq 1$, we see that $a_{i}=1$ for $i \in B_{1}$. By taking $\mathcal{J}=B_{1}$, we derive that either $\mathcal{I}_{5}^{+}=\emptyset$ or $\mathcal{I}_{5}^{-}=\emptyset$, which is a contradiction. The other case $\left(i_{7}, i_{17}\right)=(1,6)$ is excluded similarly.

Let $\left(k, Q_{0}\right)=(71,43),\left(Q_{1}, Q_{2}\right)=(53,67),\left(i_{53}, i_{67}\right)=(0,0)$. Then $\mathcal{P}_{1}=$ $\{7,11,13,19,23,71\}$. We get $\mathcal{M}_{1}=\{7,11,13,14,19,21,22,23,26,28,33,35$, $38,39,42,43,44,46,52,55,56,57,63,65,66,69,70\}, \mathcal{B}_{1}=\{1,2,3,4,5,6,8,9$, 
$10,12,15,16,17,18,20,24,25,27,29,30,31,32,34,36,37,40,41,45,47,48$, $49,50,51,54,58,59,60,61,62,64,68\}, i_{7}=i_{11}=i_{13}=i_{19}=i_{23}=0$, $i_{71}=43$. Further, for $p \in \mathcal{P}_{1}, p \nmid a_{i}$ for $i \in \mathcal{B}_{1}$. Now we take $\mathcal{P}_{0}=\mathcal{P}_{1} \cup$ $\{43,53,67\}, p_{1}=11, p_{2}=13,\left(i_{1}, i_{2}\right):=(0,0), \mathcal{I}=\mathcal{B}_{1}, \mathcal{P}=\mathcal{P}_{2}:=$ $\Lambda(11,13) \backslash \mathcal{P}_{0}=\{5,17,29,31,37,47,59,61\}$ and $\ell=\ell_{2}=\sum_{p \in \mathcal{P}_{2}}\lceil k / p\rceil$. By Lemma 5 , we see that $M=\{5,10,15,17,20,29,30,31,34,37,40,45,47,51$, $58,59,60,61,62,68\}$ is covered by $\mathcal{P}_{2}$ and $\mathfrak{i}\left(\mathcal{P}_{2}\right)$ is even for $i \in B=\{1,2,3$, $4,6,8,9,12,16,18,24,25,27,32,36,41,48,49,50,54,64\}$. We get $i_{5}=i_{17}=$ $i_{29}=i_{31}=0$, and $\{37,47,59,61\}$ is covered by $\{37,47,59,61\}$. Thus $37 \cdot 47 \cdot 59 \cdot 61 \mid a_{37} a_{47} a_{59} a_{61}$. Further, $p \nmid a_{i}$ for $i \in B$ and $p \in \mathcal{P}_{2}$. We take $\mathcal{P}_{0}=\mathcal{P}_{1} \cup \mathcal{P}_{2} \cup\{43,53,67\}, p_{1}=5, p_{2}=11,\left(i_{1}, i_{2}\right):=(0,0), \mathcal{I}=\mathcal{B}_{2}$, $\mathcal{P}=\mathcal{P}_{3}:=\Lambda(5,11) \backslash \mathcal{P}_{0}=\{3,41\}$ and $\ell=\ell_{3}=\sum_{p \in \mathcal{P}_{3}}\lceil k / p\rceil$. By Lemma 5 , we see that $M_{1}=\{3,6,12,24,27,41,48,54\}$ is covered by $\mathcal{P}_{3}$ and $\mathfrak{i}\left(\mathcal{P}_{3}\right)$ is even for $i \in B_{1}=\{1,2,4,8,9,16,18,32,36,49,64\}$. Thus $i_{3}=0$, implying $i_{41}=0$ and $p=2$ whenever $p \mid a_{i}$ for $i \in B_{1}$. By taking $\mathcal{J}=B_{1}$, we have $B_{1}=\mathcal{I}_{5}^{+} \cup \mathcal{I}_{5}^{-}$with

$$
\mathcal{I}_{5}^{+}=\{1,4,9,16,36,49,64\}, \quad \mathcal{I}_{5}^{-}=\{2,8,18,32\} .
$$

Thus $a_{i}=1$ for $i \in \mathcal{I}_{5}^{+}$and $a_{i}=2$ for $i \in \mathcal{I}_{5}^{-}$since $a_{i} \in\{1,2\}$ for $i \in B_{1}$. This is a contradiction since $43 \mid d,\left(\frac{a_{i}}{43}\right)=\left(\frac{n}{43}\right)$ and $\left(\frac{1}{43}\right) \neq\left(\frac{2}{43}\right)$.

Let $k=89, Q_{0}=79,\left(Q_{1}, Q_{2}\right)=(23,73),\left(i_{23}, i_{73}\right)=(19,15)$. Then $\mathcal{P}_{1}=$ $\{13,19,29,31,37,47,59,61,67,79,89\}$. We find that $\mathcal{M}_{1}=\{1,9,10,12,14$, $21,23,26,27,29,30,31,36,41,49,50,51,57,59,62,69,75\}, \mathcal{B}_{1}=\{0,2,3,4$, $5,6,7,8,11,13,16,17,18,20,22,24,25,28,32,33,34,35,37,38,39,40,43$, $44,45,46,47,48,52,53,54,55,56,58,60,61,63,64,66,67,68,70,71,72,73$, $74,76,77,78,79,80,81,82,83,84,85,86,87\}, i_{13}=10, i_{19}=12, i_{29}=1$, $i_{31}=26, i_{37}=14$ and $\{9,21,27,29,41\}$ is covered by $\{47,59,61,67,89\}$. Thus $i_{89} \in\{9,21,27,29,41\}$. Further, for $p \in \mathcal{P}_{1}, p \nmid a_{i}$ for $i \in \mathcal{B}_{1}$. Now we take $\mathcal{P}_{0}=\mathcal{P}_{1} \cup\{23,73,79\}, p_{1}=19, p_{2}=29,\left(i_{1}, i_{2}\right):=(12,1)$, $\mathcal{I}=\mathcal{B}_{1}, \mathcal{P}=\mathcal{P}_{2}:=\Lambda(19,29) \backslash \mathcal{P}_{0}=\{11,17,43,53,71\}$ and $\ell=\ell_{2}=$ $\sum_{p \in \mathcal{P}_{2}}\lceil k / p\rceil=22$. Thus $\left|\mathcal{I}^{\prime}\right|=|\mathcal{I}|=\left|\mathcal{B}_{1}\right|>2 \ell_{2}$. By Corollary 1 , we have $\mathcal{M}=: \mathcal{M}_{2}, \mathcal{B}=: \mathcal{B}_{2}$ such that $\left(\mathcal{M}_{2}, \mathcal{B}_{2}, \mathcal{P}_{2}, \ell_{2}\right)$ has Property $\mathfrak{H}$. We get $\mathcal{M}_{2}=$ $\{0,2,3,11,17,20,22,33,35,37,44,45,54,55,66,71,77\}, \mathcal{B}_{2}=\{4,5,6,7,8$, $13,16,18,24,25,28,32,34,38,39,40,43,46,47,48,52,53,56,58,60,61,63$, $64,67,68,70,72,73,74,76,78,79,80,81,82,83,84,85,86,87\}, i_{11}=0$, $i_{17}=3, i_{43}=2$ and $\{17,35\}$ is covered by $\{53,71\}$. Further, $p \nmid a_{i}$ for $i \in \mathcal{B}_{2}$ and $p \in \mathcal{P}_{2}$. We take $\mathcal{P}_{0}=\mathcal{P}_{1} \cup \mathcal{P}_{2} \cup\{23,73,79\}, p_{1}=11, p_{2}=13,\left(i_{1}, i_{2}\right):=$ $(0,10), \mathcal{I}=\mathcal{B}_{2}, \mathcal{P}=\mathcal{P}_{3}:=\Lambda(11,13) \backslash \mathcal{P}_{0}=\{5\}$ and $\ell=\ell_{3}=\sum_{p \in \mathcal{P}_{2}}\lceil k / p\rceil$ $=18$. Thus $\left|\mathcal{I}^{\prime}\right|=|\mathcal{I}|=\left|\mathcal{B}_{2}\right|>2 \ell_{3}$. Then the conditions of Corollary 1 are satisfied and we have $\mathcal{M}=: \mathcal{M}_{3}, \mathcal{B}=: \mathcal{B}_{3}$ with $\left(\mathcal{M}_{3}, \mathcal{B}_{3}, \mathcal{P}_{3}, \ell_{3}\right)$ having Property $\mathfrak{H}$. We get $\mathcal{M}_{3}=\{8,18,28,43,48,53,58,68,73,78,83\}, \mathcal{B}_{3}=\{4,5,6,7$, $13,16,24,25,32,34,38,39,40,46,47,52,56,60,61,63,64,67,70,72,74,76$, 
$79,80,81,82,84,85,86,87\}, i_{5}=3$. Lastly, we take $\mathcal{P}_{0}=\mathcal{P}_{1} \cup \mathcal{P}_{2} \cup \mathcal{P}_{3} \cup$ $\{23,73,79\}, p_{1}=5, p_{2}=11,\left(i_{1}, i_{2}\right):=(3,0), \mathcal{I}=\mathcal{B}_{3}, \mathcal{P}=\mathcal{P}_{4}:=\Lambda(5,11) \backslash$ $\mathcal{P}_{0}=\{3,41\}$ and $\ell=\ell_{4}=\sum_{p \in \mathcal{P}_{4}}\lceil k / p\rceil$. By Lemma 5 , we see that $M=$ $\{4,6,34,40,46,47,61,64,67,76,82,85\}$ is covered by $\mathcal{P}_{4}$ and $\mathfrak{i}\left(\mathcal{P}_{4}\right)$ is even for $i \in B=\{5,7,16,24,25,32,39,52,56,60,70,72,74,79,80,81,84,86,87\}$. Thus $i_{3}=1, i_{41}=6$ and $p \in\{2,7,83\}$ whenever $p \mid a_{i}$ for $i \in B$. Since $79 \mid d$, we see that $a_{i} \in\{1,2,83,2 \cdot 83\}$ or $a_{i} \in\{7,14,7 \cdot 83,14 \cdot 83\}$ for $i \in B$. The latter possibility is excluded since $7 \nmid i-i^{\prime}$ for all $i, i^{\prime} \in B$. By taking $\mathcal{J}=B$, we have $B=\mathcal{I}_{5}^{+} \cup \mathcal{I}_{5}^{-}$with

$$
\begin{aligned}
& \mathcal{I}_{5}^{+}=\{7,24,32,39,52,72,74,79,84,87\}, \\
& \mathcal{I}_{5}^{-}=\{5,16,25,56,60,70,80,81,86\} .
\end{aligned}
$$

Then we observe that either $a_{i} \in\{1,2 \cdot 83\}$ for $i \in \mathcal{I}_{5}^{+}$and $a_{i} \in\{2,83\}$ for $i \in \mathcal{I}_{5}^{-}$or vice versa. This is not possible by parity argument. The other case $\left(i_{23}, i_{73}\right)=(0,0)$ is excluded similarly.

5. Proof of Lemma 8. Let $7 \leq k \leq 97$ be primes. Suppose that the assumptions of Lemma 8 are satisfied. Assume that $q_{1} \mid d$ or $q_{2} \mid d$ and we shall arrive at a contradiction. We divide the proof into Subsections 5.1 and 5.2.

5.1. The cases $7 \leq k \leq 23$. We take $q=5$ in (7) and (8). We may suppose that $5 \mid d$ if $k=7,11$ and $11 \mid d$ if $k=13$. Let $5 \mid d$. Then

$$
S \subseteq\{1,6\} \quad \text { or } S \subseteq\{2,3\}
$$

according as $\left(\frac{n}{5}\right)=1$ or -1 , respectively. Thus (24) holds if $k=7,11$. Let $11 \mid d$. Then

$$
S \subseteq\{1,3,5,15\} \quad \text { or } \quad S \subseteq\{2,6,10,30\}
$$

according as $\left(\frac{n}{11}\right)=1$ or -1 , respectively. Let $13 \mid d$. Then

$$
S \subseteq\{1,3,10,30\} \quad \text { or } \quad S \subseteq\{2,5,6,15\}
$$

according as $\left(\frac{n}{13}\right)=1$ or -1 , respectively. Thus either (25) or (26) holds if $13 \leq k \leq 23$.

By observing that $a_{i}$ 's divisible by a prime $p$ can occur in at most $\lceil k / p\rceil$ terms, we have

$$
\left|T_{1}\right| \leq t_{1}^{\prime}:= \begin{cases}\sum_{p>5}\lceil k / p\rceil & \text { if } k=7,11, \\ \sum_{p>5}\lceil k / p\rceil-2 & \text { if } 13 \leq k<23, \\ \sum_{p>5}\lceil k / p\rceil-3 & \text { if } k=23,\end{cases}
$$

where the sum is taken over all $p \leq k$. For the last sum, we observe that 7 and 11 together divide at most six $a_{i}$ 's when $k=23$. We divide the proof into four cases. 
CASE I. Let $2 \nmid d$ and $3 \nmid d$. From (24)-(26), (10) and Lemma 1, we get $|T| \leq t_{1}:=\left\{\begin{array}{r}\max \left(f_{1}(k, 1,0)+f_{1}(k, 6,0), f_{1}(k, 2,0)+f_{1}(k, 3,0)\right)+\lceil k / 4\rceil \\ \text { if } k=7,11 \\ f_{1}(k, 1,0)+f_{1}(k, 3,0)+f_{1}(k, 5,0)+f_{1}(k, 15,0)+\lceil k / 4\rceil \\ \text { if } k>11,\end{array}\right.$

since $f_{1}(k, a, \delta)$ is a nonincreasing function of $a$ and $\sum_{a \in R} \nu_{\mathrm{e}}(a) \leq\lceil k / 4\rceil$. We check that $k=|T|+\left|T_{1}\right| \leq t_{1}+t_{1}^{\prime}<k$, a contradiction.

Thus we have either $2 \mid d$ or $3 \mid d$. Let $k=7,11$. If $2 \mid d$, then $S \subseteq\{1\}$ or $S \subseteq\{3\}$. If $3 \mid d$, we have $S \subseteq\{1\}$ or $S \subseteq\{2\}$. By Lemma 2 , we get $|T| \leq(k-1) / 2$. We check that $k=|T|+\left|T_{1}\right| \leq(k-1) / 2+t_{1}^{\prime}<k$ by $(27)$. This is a contradiction. From now on, we may also suppose that $13 \leq k \leq 23$.

CASE II. Let $2 \mid d$ and $3 \nmid d$. Then $S \subseteq\{1,3,5,15\}$ if $11 \mid d$ and $S \subseteq\{1,3\}$ or $S \subseteq\{5,15\}$ if $13 \mid d$. Let $2 \| d$. From (10) and Lemma 1 with $\delta=1$, we get

$$
|T| \leq F(k, 1,1)+F(k, 3,1)+F(k, 5,1)+F(k, 15,1)=: t_{2} .
$$

Let $4 \| d$. From $a_{i} \equiv n(\bmod 4)$, we see that $S \subseteq\{1,5\}$ or $S \subseteq\{3,15\}$ if $11 \mid d$, and either $S=\emptyset$ or $S=\{1\},\{3\},\{5\}$ or $\{15\}$ if $13 \mid d$. Therefore

$$
|T| \leq F(k, 1,2)+F(k, 5,2)=: t_{3}
$$

by Lemma 1 with $\delta=2$. Let $8 \mid d$. Then $a_{i} \equiv n(\bmod 8)$ and Lemma 1 with $\delta=3$ imply

$$
|T| \leq F(k, 1,3)=: t_{4} .
$$

Thus $|T| \leq \max \left(t_{2}, t_{3}, t_{4}\right)$. This with (27) contradicts (9).

CASE III. Let $2 \nmid d$ and $3 \mid d$. From $a_{i} \equiv n(\bmod 3)$, we see that either $S=\emptyset$ or $S=\{1\},\{2\},\{5\}$ or $\{10\}$ if $11 \mid d$, and $S \subseteq\{1,10\}$ or $S \subseteq\{2,5\}$ if $13 \mid d$. By (10) and Lemma 1, we get

$$
|T| \leq F(k, 1,0)+F(k, 5,0),
$$

which together with (27) contradicts (9).

CASE IV. Let $2 \mid d$ and $3 \mid d$. Then $S \subseteq\{1\},\{5\}$. By Lemma 2, we get $|T| \leq(k-1) / 2$. We check that $k=|T|+\left|T_{1}\right| \leq(k-1) / 2+t_{1}^{\prime}<k$, a contradiction.

5.2. The cases $k \geq 29$. Let $29 \leq k \leq 59$ and $19 \mid d$. Then by Lemma 7 with $Q_{0}=19$, we get $7 \mid d$ or $17 \mid d$. Thus we get a prime pair $\left(Q, Q^{\prime}\right)=(7,19)$ or $\left(Q, Q^{\prime}\right)=(17,19)$ such that $Q Q^{\prime} \mid d$. Similarly we get $\left(Q, Q^{\prime}\right)=(7,29)$ or $\left(Q, Q^{\prime}\right)=(17,29)$ with $Q Q^{\prime} \mid d$ when $31 \leq k \leq 59$ and $29 \mid d$. Let $k=71$. Then we have either $43|d, 67| d$ or $43 \mid d, 67 \nmid d$ or $43 \nmid d, 67 \mid d$. We get a prime pair $\left(Q, Q^{\prime}\right)=(43,67)$ with $Q Q^{\prime} \mid d$ if $43|d, 67| d$. If $43 \mid d, 67 \nmid d$, we deduce from Lemma 7 with $Q_{0}=43$ that $53 \mid d$ and we take $\left(Q, Q^{\prime}\right)=(43,53)$ such 
that $Q Q^{\prime} \mid d$. If $43 \nmid d, 67 \mid d$, we find from Lemma 7 with $Q_{0}=67$ that $53 \mid d$ and we take $\left(Q, Q^{\prime}\right)=(53,67)$ such that $Q Q^{\prime} \mid d$. Similar prime pairs $\left(Q, Q^{\prime}\right)$ with $Q Q^{\prime} \mid d$ for each $61 \leq k \leq 97$ are given in the table below. For $q \leq 17$, we see that

$$
\left|T_{1}\right| \leq \sum_{\substack{p>q \\ p \neq Q, Q^{\prime}}}\left\lceil\frac{k}{p}\right\rceil \leq t_{2}^{\prime}:= \begin{cases}\sum_{p>q}\lceil k / p\rceil-2 & \text { if } 29 \leq k \leq 61, \\ \sum_{p>q}\lceil k / p\rceil-4 & \text { if } 61<k<97, \\ \sum_{p>q}\lceil k / p\rceil-7 & \text { if } k=97,\end{cases}
$$

where the sum is taken over primes $\leq k$.

\begin{tabular}{ccc}
\hline$k$ & $\left(Q, Q^{\prime}\right)$ & $S \subseteq S^{\prime}$ with $S^{\prime}$ given by one of \\
\hline $29 \leq k \leq 59$ & $(7,19),(7,29)$ & $\{1,30\},\{2,15\},\{3,10\},\{5,6\}$ \\
$29 \leq k \leq 59$ & $(17,19),(17,29)$ & $\{1,30,35,42\},\{2,15,21,70\},\{3,10,14,105\},\{5,6,7,210\}$ \\
61 & $(11,59)$ & $\{1,3,5,15\},\{2,6,10,30\},\{7,21,35,105\},\{14,42,70,210\}$ \\
67,71 & $(43,53)$ & $\{1,6,10,15\},\{2,3,5,30\},\{7,42,70,105\},\{14,21,35,210\}$ \\
71 & $(43,67)$ & See $(29)$ \\
71 & $(53,67)$ & $\{1,6,10,15\},\{2,3,5,30\},\{7,42,70,105\},\{14,21,35,210\}$ \\
73 & $(23,53)$ & $\{1,6,70,105\},\{2,3,35,210\},\{5,14,21,30\},\{7,10,15,42\}$ \\
73 & $(23,67)$ & $\{1,6,35,210\},\{2,3,70,105\},\{5,7,30,42\},\{10,14,15,21\}$ \\
79 & $(23,53),(53,73)$ & $\{1,6,70,105\},\{2,3,35,210\},\{5,14,21,30\},\{7,10,15,42\}$ \\
79 & $(23,67),(67,73)$ & $\{1,6,35,210\},\{2,3,70,105\},\{5,7,30,42\},\{10,14,15,21\}$ \\
83 & $(23,37),(37,73)$ & $\{1,3,70,210\},\{2,6,35,105\},\{5,14,15,42\},\{7,10,21,30\}$ \\
89 & $(23,79),(73,79)$ & $\{1,2,105,210\},\{3,6,35,70\},\{5,10,21,42\},\{7,14,15,30\}$ \\
97 & $(23,37),(23,83)$ & $\{1,3,70,210\},\{2,6,35,105\},\{5,14,15,42\},\{7,10,21,30\}$ \\
\hline
\end{tabular}

CASE I. Let $2 \nmid d$ and $3 \nmid d$. In (7) and (8) we take $q=11$ if $k=71$, $\left(Q, Q^{\prime}\right)=(43,67)$ and $q=7$ otherwise. From $\left(\frac{a_{i}}{Q}\right)=\left(\frac{n}{Q}\right)$ and $\left(\frac{a_{i}}{Q^{\prime}}\right)=\left(\frac{n}{Q^{\prime}}\right)$, we get $S \subseteq S^{\prime}=\left\{s: s\right.$ squarefree, $\left.P(s) \leq q,\left(\frac{s}{Q}\right)=\left(\frac{n}{Q}\right),\left(\frac{s}{Q^{\prime}}\right)=\left(\frac{n}{Q^{\prime}}\right)\right\}$. By considering $\left(\left(\frac{n}{Q}\right),\left(\frac{n}{Q^{\prime}}\right)\right)=(1,1),(1,-1),(-1,1)$ and $(-1,-1)$, we get four possibilities for $S^{\prime}$. For each value of $k$, the above table shows $\left(Q, Q^{\prime}\right)$ and $S^{\prime}$. For $k=71,\left(Q, Q^{\prime}\right)=(43,67)$, we get $S \subseteq S^{\prime}$ with $S^{\prime}$ given by one of

$$
\{1,6,10,14,15,21,35,210\},\{2,3,5,7,30,42,70,105\} \text {, }
$$

$\{11,66,110,154,165,231,385,2310\},\{22,33,55,77,330,462,770,1155\}$.

From the possibilities for $S \subseteq S^{\prime}$ given by the table, (10) and Lemma 1 , we get

$$
|T| \leq t_{5}:=\max \sum_{s \in S^{\prime}} F(k, s, 0)
$$

where the maximum is taken over all the four choices of $S^{\prime}$. This with (28) gives $|T|+\left|T_{1}\right| \leq t_{5}+t_{2}^{\prime}<k$, contradicting (9). 
CASE II. Let $2 \mid d$ and $3 \nmid d$. We take $q=7$ for $2\|d, 4\| d$ and $q=11$ for $8 \mid d$.

Let $2 \| d$. Then $S \subseteq\{1,3,5,7,15,21,35,105\}=: S_{2}$. From (10) and Lemma 1 with $\delta=1$, we get

$$
|T| \leq \sum_{s \in S_{2}} F(k, s, 1)=: t_{6}
$$

Let $4 \| d$. Then we see that either $S \subseteq\{1,5,21,105\}=: S_{41}$ or $S \subseteq\{3,7,15,35\}$ $=: S_{42}$. From (10) and Lemma 1 with $\delta=2$, we get

$$
|T| \leq \max _{i=1,2} \sum_{s \in S_{4 i}} F(k, s, 2)=: t_{7} .
$$

Hence, if $8 \nmid d$, then $|T| \leq \max \left(t_{6}, t_{7}\right)$. This with (28) implies $|T|+\left|T_{1}\right| \leq$ $\max \left(t_{6}, t_{7}\right)+t_{2}^{\prime}<k$, contradicting $(9)$.

Let $8 \mid d$. Then we see from $a_{i} \equiv n(\bmod 8)$ that $S \subseteq\{1,33,105,385\}=$ : $S_{81}$ or $S \subseteq\{3,11,35,1155\}=: S_{82}$ or $S \subseteq\{5,21,77,165\}=: S_{83}$ or $S \subseteq$ $\{7,15,55,231\}=: S_{84}$. Then

$$
|T| \leq \max _{1 \leq i \leq 4} \sum_{s \in S_{8 i}} F(k, s, 3)=: t_{8}
$$

by Lemma 1 with $\delta=3$. This with (28) implies $|T|+\left|T_{1}\right| \leq t_{8}+t_{2}^{\prime}<k$, a contradiction.

CASE III. Let $2 \nmid d$ and $3 \mid d$. We take $q=11$. Then by modulo 3 , we get either $S \subseteq\{1,7,10,22,55,70,154,385\}=: S_{31}$ or $S \subseteq\{2,5,11,14,35,77$, $110,770\}=: S_{32}$. By (10) and Lemma 1, we get

$$
|T| \leq \max _{i=1,2} \sum_{s \in S_{3 i}} F(k, s, 0)=: t_{9} .
$$

This together with (28) contradicts (9).

CASE IV. Let $2 \mid d$ and $3 \mid d$. Let $2 \| d$. We take $q=7$. Then we see that either $S \subseteq\{1,7\}$ or $S \subseteq\{5,35\}$. By (10) and Lemma 1, we get $|T| \leq$ $F(k, 1,1)+F(k, 7,1)$, which together with (28) contradicts (9).

Let $4 \| d$. We take $q=13$. From $a_{i} \equiv n(\bmod 12)$, we see that

$$
\begin{aligned}
S \subseteq S^{\prime} \in \mathfrak{S}:= & \{\{1,13,385,5005\},\{5,65,77,1001\}, \\
& \{7,55,91,715\},\{11,35,143,455\}\} .
\end{aligned}
$$

Then

$$
|T| \leq \max _{S^{\prime} \in \mathfrak{S}} \sum_{s \in S^{\prime}} F(k, s, 2),
$$

which together with (28) contradicts (9). 
Let $8 \mid d$. We take $q=17$. From $a_{i} \equiv n(\bmod 24)$, we see that $S \subseteq S^{\prime}=$ $\{1,385,1105,17017\}$ or $S \subseteq S^{\prime \prime} \in \mathfrak{S}_{1}$ where $\mathfrak{S}_{1}$ is the union of sets

$\{5,77,221,85085\},\{7,55,2431,7735\}$,

$\{11,35,1547,12155\},\{13,85,1309,5005\},\{17,65,1001,6545\}$,

$\{91,187,595,715\},\{119,143,455,935\}$.

Let $S \subseteq S^{\prime \prime} \in \mathfrak{S}_{1}$. Then

$$
|T| \leq \max _{S^{\prime \prime} \in \mathfrak{S}_{1}} \sum_{s \in S^{\prime \prime}} F(k, s, 3)=: t_{10}
$$

Let $S \subseteq S^{\prime}$. By Lemma 2 , we get $\nu(1) \leq(k-1) / 2$. This together with $\nu(1105)+\nu(17017) \leq 1$ by $13 \cdot 17 \mid \operatorname{gcd}(1105,17017)$ and $\nu(385) \leq 1$ by Lemma 1 gives $|T| \leq(k-1) / 2+2$. Therefore $|T| \leq \max \left(t_{10},(k-1) / 2+2\right)$, which with (28) contradicts (9).

6. Proof of Theorem 4. Let $k=7$. By the case $k=6$, we may assume that $7 \nmid d$. Now the assertion follows from Lemmas 8 and 6 . Let $k=8$. Then by applying the case $k=7$ twice to $n(n+d) \cdots(n+6 d)=b^{\prime} y^{\prime 2}$ and $(n+d) \cdots(n+7 d)=b^{\prime \prime} y^{\prime \prime 2}$, we get

$$
\begin{array}{r}
\left(a_{0}, \ldots, a_{6}\right),\left(a_{1}, \ldots, a_{7}\right) \\
\in\{(2,3,1,5,6,7,2),(3,1,5,6,7,2,1),(1,5,6,7,2,1,10), \\
(2,7,6,5,1,3,2),(1,2,7,6,5,1,3),(10,1,2,7,6,5,1)\} .
\end{array}
$$

This gives $\left(a_{0}, \ldots, a_{7}\right)=(2,3,1,5,6,7,2,1),(3,1,5,6,7,2,1,10)$ or their mirror images and the assertion follows. Let $k=9$. By applying the case $k=8$ twice to $n(n+d) \cdots(n+7 d)=b^{\prime} y^{\prime 2}$ and $(n+d) \cdots(n+8 d)=$ $b^{\prime \prime} y^{\prime \prime 2}$, we get the result. Let $k=10$. By applying $k=9$ twice, we get $\left(a_{0}, a_{1}, \ldots, a_{8}\right),\left(a_{1}, a_{2}, \ldots, a_{8}, a_{9}\right) \in\{(2,3, \ldots, 1,10),(10,1, \ldots, 3,2)\}$, which is not possible.

Let $k \geq 11$ and $k^{\prime}<k$ be consecutive primes. We suppose that Theorem 4 is valid with $k$ replaced by $k^{\prime}$. Let $k \mid d$. Then $\left(\frac{a_{i}}{k}\right)=\left(\frac{n}{k}\right)$ for all $0 \leq i<k$. By applying the case $k=k^{\prime}$ to $n(n+d) \cdots\left(n+\left(k^{\prime}-1\right) d\right)=b^{\prime} y^{\prime 2}$ with $P\left(b^{\prime}\right) \leq k^{\prime}$, we get $k^{\prime} \leq 23$ and $1,2,3,5 \in\left\{a_{0}, a_{1}, \ldots, a_{k^{\prime}-1}\right\}$ in view of (5) and (6). Therefore $\left(\frac{2}{k}\right)=\left(\frac{3}{k}\right)=\left(\frac{5}{k}\right)=1$, which is not possible.

Thus we may assume that $k \nmid d$ and $k \mid n+i d$ for some $0 \leq i \leq(k-1) / 2$ by considering the mirror image (4) of (2) whenever Theorem 4 holds at $k^{\prime}$. We shall use this assertion without reference in the proof of Theorem 4.

Let $k=11$. By Lemmas 8 and 6 , we see that $11 \mid n+i d$ for $0 \leq i \leq 3$. If $11 \mid n$, the assertion follows by the case $k=10$. Let $11 \mid n+d$. We consider $(n+2 d) \cdots(n+10 d)=b^{\prime} y^{\prime 2}$ with $P\left(b^{\prime}\right) \leq 7$ and the case $k=9$ to get $\left(a_{2}, a_{3}, \ldots, a_{10}\right) \in\{(2,3,1,5,6,7,2,1,10),(10,1,2,7,6,5,1,3,2)\}$. The first 
possibility is excluded since $1=\left(\frac{14}{11}\right)=\left(\frac{a_{2} a_{7}}{11}\right)=\left(\frac{1 \cdot 6}{11}\right)=-1$. For the second possibility, we observe $P\left(a_{0}\right) \leq 5$ since $\operatorname{gcd}\left(a_{0}, 7 \cdot 11\right)=1$ and this is excluded by the case $k=6$ applied to $n(n+2 d)(n+4 d)(n+6 d)(n+8 d)$. $(n+10 d)$. Let $11 \mid n+2 d$. Then by the case $k=8$, we have $\left(a_{3}, a_{4}, \ldots, a_{10}\right)$ $\in\{(2,3,1,5,6,7,2,1),(3,1,5,6,7,2,1,10),(1,2,7,6,5,1,3,2),(10,1,2,7,6$, $5,1,3)\}$. The first three possibilities are excluded by considering the values of the Legendre symbol mod 11 at $a_{3}, a_{8}$, at $a_{3}, a_{4}$ and at $a_{3}, a_{5}$, respectively. If the last possibility holds, then $a_{0}=1 \operatorname{since} \operatorname{gcd}\left(a_{0}, 2 \cdot 3 \cdot 5 \cdot 7 \cdot 11\right)=1$, and this is not possible since $1=\left(\frac{a_{0} a_{4}}{11}\right)=\left(\frac{(-2) 2}{11}\right)=-1$. Let $11 \mid n+3 d$. We consider $(n+4 d) \cdots(n+10 d)=b^{\prime} y^{\prime 2}$ with $P\left(b^{\prime}\right) \leq 7$ and the case $k=7$ to infer that $\left(a_{4}, \ldots, a_{10}\right) \in\{(2,3,1,5,6,7,2),(3,1,5,6,7,2,1),(1,5,6,7,2,1,10)$, $(2,7,6,5,1,3,2),(1,2,7,6,5,1,3),(10,1,2,7,6,5,1)\}$, which is not possible as above. This completes the proof for $k=11$. The assertion for $k=12$ follows from that of $k=11$.

Let $k=13$. Then the assertion follows from Lemmas 8,6 and the case $k=11$. Let $k=14$. By applying the case $k=13$ to $n(n+d) \cdots(n+12 d)=$ $b^{\prime} y^{\prime 2}$ and $(n+d) \cdots(n+13)=b^{\prime \prime} y^{\prime \prime 2}$, we get the assertion. Let $k=15$. Then applying the case $k=14$ both to $n(n+d) \cdots(n+13 d)$ and $(n+d) \cdots(n+14 d)$ gives the result. For $k=16$ the assertion follows from the case $k=15$.

Let $k=17$. Then $17 \mid n+2 d$ or $17 \mid n+3 d$ by Lemmas 8,6 and the case $k=15$. Let $17 \mid n+2 d$. Then by applying the case $k=14$ to $(n+3 d) \cdots$ $(n+16 d)=b^{\prime} y^{\prime 2}$ with $P\left(b^{\prime}\right) \leq 13$, we get $\left(a_{3}, a_{4}, \ldots, a_{16}\right) \in\{(3,1, \ldots, 15,1)$, $(1,15, \ldots, 1,3)\}$. The first possibility is excluded by considering the Legendre symbol mod 17 at $a_{3}, a_{4}$. For the second, we observe that $\operatorname{gcd}\left(a_{1}, 7 \cdot 11\right.$. $13 \cdot 17)=1$, which is not possible by the case $k=6$ applied to $(n+d)$. $(n+4 d)(n+7 d)(n+10 d)(n+13 d)(n+16 d)$. Let $17 \mid n+3 d$. By considering $(n+4 d) \cdots(n+16 d)=b^{\prime} y^{\prime 2}$ with $P\left(b^{\prime}\right) \leq 13$, it follows from the case $k$ $=13$ that $\left(a_{4}, \ldots, a_{16}\right) \in\{(3,1, \ldots, 14,15),(1,5, \ldots, 15,1),(15,14, \ldots, 1,3)$, $(1,15, \ldots, 5,1)\}$. The first three possibilities are excluded by considering the Legendre symbol mod 17 at $a_{4}, a_{5}$. If the last possibility holds, we observe that $a_{1}=1$ since $\operatorname{gcd}\left(a_{1}, \prod_{p \leq 17} p\right)=1$ and then $1=\left(\frac{a_{1} a_{4}}{17}\right)=\left(\frac{(-6)(-3)}{17}\right)=$ -1 , a contradiction. The assertion for $k=18$ follows from that for $k=17$.

Let $k=19$. Then the assertion follows from Lemmas 8,6 and the case $k=17$. By applying the case $k=19$ twice to $n(n+d) \cdots(n+18 d)$ and $(n+d) \cdots(n+18 d)(n+19 d)$, the assertion for $k=20$ follows and this also implies the cases $k=21,22$.

Let $k=23$. We see from Lemmas 8, 6 and the case $k=20$ that 23 divides $n+3 d$. We consider the case $k=19$ and $(n+4 d) \cdots(n+22 d)=b^{\prime} y^{\prime 2}$ with $P\left(b^{\prime}\right) \leq 19$ to get $\left(a_{4}, a_{5}, \ldots, a_{22}\right)=(1,5, \ldots, 21,22)$ or $(22,21, \ldots, 5,1)$. By considering the values of the Legendre symbol mod 23 at $a_{4}$ and $a_{5}$, we may assume the second possibility. Now $P\left(a_{2}\right) \leq 11$ and this is not possible by 
the case $k=11$ applied to $(n+2 d)(n+4 d) \cdots(n+22 d)$. Let $k=24$. We get $\left(a_{0}, a_{1}, \ldots, a_{23}\right)=(5,6, \ldots, 3,7)$ or $(7,3, \ldots, 6,5)$ by applying the case $k=23$ both to $n(n+d) \cdots(n+22 d)$ and $(n+d) \cdots(n+23 d)$. Further, the assertion for $25 \leq k \leq 28$ follows from $k=24$.

Let $k \geq 29$. First we consider $k=29$. We see from Lemmas 8,6 and the case $k=25$ that $29 \mid n+4 d$ or $29 \mid n+5 d$. Let $29 \mid n+4 d$. Then applying the case $k=24$ to $(n+5 d)(n+6 d) \cdots(n+28 d)$, we get $\left(a_{5}, a_{6}, \ldots, a_{28}\right)=$ $(5,6, \ldots, 3,7)$ or $(7,3, \ldots, 6,5)$. By observing $1=\left(\frac{30}{29}\right)=\left(\frac{a_{5} a_{6}}{29}\right)=\left(\frac{1 \cdot 2}{29}\right)=$ -1 , we may assume the second possibility. Then $a_{1}=1$, implying $1=$ $\left(\frac{a_{2} a_{8}}{29}\right)=\left(\frac{(-2) 4}{29}\right)=-1$, a contradiction. Let $29 \mid n+5 d$. Now by considering $k=23$ and $(n+6 d) \ldots(n+28 d)$, we get $\left(a_{6}, a_{7}, \ldots, a_{28}\right) \in\{(5,6, \ldots, 26,3)$, $(6,7, \ldots, 3,7),(3,26, \ldots, 6,5),(7,3, \ldots, 7,6)\}$. Then we may restrict to the last possibility by considering the Legendre symbol mod 29 at the first two entries in the remaining possibilities. It follows that $a_{3}=1$, implying $1=$ $\left(\frac{a_{3} a_{9}}{29}\right)=\left(\frac{(-2) 4}{29}\right)=-1$, a contradiction. This completes the proof for $k=29$. We now proceed by induction. By Lemmas 8 and 6 , the assertion follows for all primes $k$. Now Lemma 3 completes the proof of Theorem 4 .

7. Proof of Theorem 1. Observe that for all tuples in (5) and (6), the product of the $a_{i}$ 's is not a square. Hence, by Theorem 4, we may assume that $101 \leq k \leq 109$. Assume (1). Then $\operatorname{ord}_{p}\left(a_{0} a_{1} \cdots a_{k-1}\right)$ is even for each prime $p$. Let $101 \leq k \leq 105$. Then $P\left(a_{4} a_{5} \cdots a_{100}\right) \leq 97$. Now the assertion follows from Theorem 4 by considering $(n+4 d) \cdots(n+100 d)$ and $k=$ 97 . Let $k=106,107$. Then $P\left(a_{4} a_{5} \cdots a_{102}\right) \leq 101$. We may suppose that $P\left(a_{4} a_{5}\right)=101$ or $P\left(a_{101} a_{102}\right)=101$, otherwise the assertion follows by the case $k=99$ in Theorem 4 . Let $P\left(a_{4} a_{5}\right)=101$. Then $P\left(a_{6} \cdots a_{102}\right) \leq 97$ and the assertion follows by the case $k=97$ in Theorem 4 . This is also true when $P\left(a_{101} a_{102}\right)=101$ since $P\left(a_{4} \cdots a_{100}\right) \leq 97$ in this case. Let $k=108,109$. Then $P\left(a_{6} \cdots a_{102}\right) \leq 101$. Thus either $P\left(a_{6} a_{7}\right)=101$ or $P\left(a_{101} a_{102}\right)=101$. Let $P\left(a_{6} a_{7}\right)=101$. Then $P\left(a_{8} \cdots a_{102}\right) \leq 97$. We may assume that $97 \mid a_{8} a_{9} a_{10} a_{11}$ or $97 \mid a_{97} \cdots a_{101} a_{102}$. Let $97 \mid a_{8} a_{9} a_{10} a_{11}$. Then $P\left(a_{12} a_{13} \cdots a_{102}\right) \leq 89$ and the assertion follows by the case $k=91$ of Theorem 4. Let $97 \mid a_{97} \cdots a_{102}$. Then $P\left(a_{8} a_{9} \cdots a_{96}\right) \leq 89$ and the assertion follows from the case $k=89$ of Theorem 4 . When $P\left(a_{101} a_{102}\right)=101$, we argue as above to get the assertion.

\section{References}

[BBGH06] M. A. Bennett, N. Bruin, K. Gyôry and L. Hajdu, Powers from products of consecutive terms in arithmetic progression, Proc. London Math. Soc. 92 (2006), 273-306. 
[BF05] N. Bruin and E. V. Flynn, Towers of 2-covers of hyperelliptic curves, Trans. Amer. Math. Soc. 357 (2005), 4329-4347.

[Erd39] P. Erdôs, Note on the product of consecutive integers (II), J. London Math. Soc. 14 (1939), 245-249.

[ES75] P. Erdôs and J. L. Selfridge, The product of consecutive integers is never a power, Illinois J. Math. 19 (1975), 292-301.

[Eul80] L. Euler, Mém. Acad. Sci. St. Petersb. 8, 1817-1818 (1780), 3; Comm. Arith. Collectae II, 411-413.

[Lai04] S. Laishram, Topics in Diophantine equations, M.Sc. thesis, Mumbai Univ., 2004; online at http://www.math.tifr.res.in/ shanta/MScthesis.pdf.

[LS] S. Laishram and T. N. Shorey, The equation $n(n+d) \cdots(n+(k-1) d)=b y^{2}$ with $\omega(d) \leq 6$ or $d \leq 10^{10}$, Acta Arith., to appear.

[Mor69] L. J. Mordell, Diophantine Equations, Academic Press, New York, 1969.

[MS03] A. Mukhopadhyay and T. N. Shorey, Almost squares in arithmetic progression (II), Acta Arith. 110 (2003), 1-14.

[Obl50] R. Obláth, Über das Produkt fünf aufeinander folgender Zahlen in einer arithmetischen Reihe, Publ. Math. Debrecen 1 (1950), 222-226.

[Rig39] O. Rigge, Über ein diophantisches Problem, in: C. R. $9^{\text {ème }}$ Congrès des Mathématiciens Scandinaves (Helsingfors, 1938), Mercator, 1939, 155-160.

[Tij89] R. Tijdeman, Diophantine equations and diophantine approximations, in: Number Theory and Applications, Kluwer, Dordrecht, 1989, 215-243.

Department of Mathematics

College of Science and Technology

Nihon University

Tokyo 101-8308, Japan

E-mail: hirata@math.cst.nihon-u.ac.jp

Mathematical Institute

Leiden University

2300 RA Leiden, The Netherlands

E-mail: tijdeman@math.leidenuniv.nl
School of Mathematics Tata Institute of Fundamental Research Homi Bhabha Road Mumbai 400 005, India E-mail: shanta@math.tifr.res.in shorey@math.tifr.res.in

Received on 2.12.2006

and in revised form on 13.4.2007 